\title{
Análisis de la morfología ósea de peces de la laguna costera Mar Chiquita, Argentina
}

\author{
Analysis of fish skeletal morphology in Mar Chiquita coastal lagoon, Argentina \\ Mariana Y. Deli Antoni1, ${ }^{1,}$, Mariano González Castro ${ }^{1}$ y Juan M. Díaz de Astarloa1, 2 \\ ${ }^{1}$ Facultad de Ciencias Exactas y Naturales, Departamento de Ciencias Marinas, Universidad Nacional de Mar del Plata. \\ Funes 3350. 7600 Mar del Plata, Argentina \\ ${ }^{2}$ Consejo Nacional de Investigaciones Científicas y Técnicas (CONICET), Av. Rivadavia 1917, Buenos Aires, Argentina \\ astarloa@mdp.edu.ar
}

\begin{abstract}
The morphological analysis of the bones is a valuable tool for taxonomy, which has allowed the definition of the higher groups of vertebrates and descriptions and identifications of fish species. Therefore, the compared osteology provides valuable diagnostic elements in biodiversity studies, ecology and dynamics of trophic habits of piscivore fish. The goal of the present study is to make a descriptive and comparative osteological analysis that allow the identification of the main fish species occurring in Mar Chiquita coastal lagoon: the brazilian menhaden (Brevoortia aurea), the mullet (Mugil platanus), the silver side (Odontesthes argentinensis), the white croaker (Micropogonias furnieri), the black drum (Pogonias cromis), the flounder (Paralichthys orbignyanus), the blue fish (Pomatomus saltatrix), the stripped weakfish (Cynoscion guatucupa) and the southern kingfish (Menticirrhus americanus). Six specimens of each species were analyzed and methods to disarticulate skeletons in fresh and frozen specimens were used. On the basis of the diagnostic characteristics of the bones and their degree of digestibility resistance, eleven bones were selected: parasphenoid, lachrymal, premaxilla, dentary, anguloarticular, palatine, hyomandibular, ceratohyal, urohyal, preopercle and posttemporal. Each bone has been described, and according to the most important differences found among fish species, identification keys were elaborated. We conclude that bone morphology constitutes a valuable diagnostic tool for the identification of fish species occurring in Mar Chiquita coastal lagoon and coastal adjacent areas.
\end{abstract}

Key words: Osteology, taxonomy, Mar Chiquita coastal lagoon

\section{Introducción}

Uno de los objetivos más importantes de los estudios biológicos en la actualidad, es el de suministrar elementos de juicio útiles para establecer pautas tendientes a preservar la biodiversidad. Entre los diversos aspectos que hacen al conocimiento del funcionamiento de los ecosistemas, requisito previo para cualquier intento de manejo conservacionista, es de suma importancia
Resumen.- El estudio morfológico de los elementos óseos es una herramienta valiosa para la taxonomía, que ha permitido la definición de los grandes grupos de vertebrados y la descripción e identificación de especies de peces. Así, la osteología comparada aporta elementos diagnósticos utilizables en estudios de biodiversidad, ecología trófica y dinámica de la alimentación de organismos ictiófagos. El objetivo del presente trabajo es realizar un análisis osteológico descriptivo y comparativo que permita la identificación, a través de los elementos óseos, de las principales especies de peces presentes en Mar Chiquita: la saraca (Brevoortia aurea), la lisa (Mugil platanus), el pejerrey de mar (Odontesthes argentinensis), la corvina rubia (Micropogonias furnieri), la corvina negra (Pogonias cromis), el lenguado de fango (Paralichthys orbignyanus), la anchoa de banco (Pomatomus saltatrix), la pescadilla de red (Cynoscion guatucupa) y la burriqueta (Menticirrhus americanus). Se analizaron seis ejemplares de cada especie y se utilizaron técnicas de desarticulación en peces frescos y congelados. Sobre la base de las características diagnósticas de los huesos y su grado de resistencia a la digestibilidad fueron seleccionados once elementos óseos: paraesfenoides, lacrimal, premaxilar, dentario, anguloarticular, palatino, hiomandibular, ceratohial, urohial, preopérculo y postemporal. Se realizó la descripción de los huesos mencionados y de acuerdo a las diferencias más importantes encontradas entre las especies estudiadas, se confeccionaron claves de identificación. Se concluye que la morfología de los elementos óseos constituye una herramienta diagnóstica valiosa para la determinación específica de los peces presentes en la laguna costera Mar Chiquita y zonas costeras aledañas.

Palabras clave: osteología, taxonomía, laguna costera Mar Chiquita

establecer las composiciones taxonómicas de los factores bióticos al nivel más detallado posible (Gosztonyi \& Kuba 1996). La determinación de los ítems presa hasta la menor categoría taxonómica es muy importante en estudios de ecología trófica y trofodinámica de peces, ya que no solamente aporta información sobre la diversidad de la dieta de aquellos organismos que se alimentan de peces, sino que provee valiosos conocimientos sobre la biodiversidad íctica de una determinada región y 
complementa la información obtenida por métodos tradicionales (Watt et al. 1997, Granadeiro \& Silva 2000, Favero et al. 2001).

La laguna costera Mar Chiquita $\quad\left(37^{\circ} 32^{\prime}-37^{\circ} 45^{\prime} S\right.$; $57^{\circ} 19^{\prime}-57^{\circ} 26^{\prime} \mathrm{W}$ ), se localiza a $32 \mathrm{~km}$ al noreste de la ciudad de Mar del Plata, en la Provincia de Buenos Aires, Argentina. Fue declarada Reserva Mundial de la Biosfera en 1996 y Reserva Provincial en 1999 (Iribarne 2001). Es habitada por una riquísima fauna de organismos invertebrados (insectos, anélidos poliquetos, moluscos y crustáceos) y vertebrados (peces, anfibios, reptiles, aves y mamíferos) (Bó et al. 2001, Cousseau et al. 2001, De Francesco \& Isla 2001, Ferrero 2001, Malizia et al. 2001, Spivak et al. 2001, Vega 2001, Schwindt 2003) que utilizan a la laguna como área de reproducción, engorde, abrigo y/o cría, debido a que proporciona un alto grado de protección y abundante disponibilidad de alimento. Desde el punto de vista zoogeográfico, los peces de agua dulce de Mar Chiquita forman parte de la Provincia Parano-Platense (Azpelicueta et al. 1998), pero constituyen una fauna íctica menos diversa si se la compara con la fauna típica de esa Provincia, en los espejos de agua del nordeste de Argentina.

Los estudios que aportan información sobre la biodiversidad íctica de la laguna costera Mar Chiquita se refieren a la composición específica y su relación con variables ambientales o espaciotemporales (Azpelicueta et al. 1998, Cousseau et al. 2001). Otros han sido relacionados con la alimentación de algunos peces presentes en la laguna como los lenguados (Rivera Prisco et al. 2001) y las corvinas (Hozbor \& García de la Rosa 2000), la visita ocasional de especies no frecuentes (Lucifora \& Menni 1998, Díaz de Astarloa et al. 2000, Figueroa et al. 2000, González Castro et al. 2006, Heras et al. 2006), aspectos de la distribución y estructura poblacional (Cerdá Gilbert 1999, Abruzzini 2002) y biología reproductiva (González Castro 2007).

El sistema óseo constituye el fundamento sobre el cual se apoya la definición de los grandes grupos y la discusión de afinidades y diferencias entre los mismos (Greenwood et al. 1966, Monod 1968, Greenwood et al. 1973, Nybelin 1973, Rosen 1973, Cervigón 1980). De esta manera, la osteología de peces ha permitido realizar estudios filogenéticos (Pietsch \& Zabetian 1990, Collette \& Gillis 1992, Chapleau 1993, Hoshino 2001), aportar elementos para la descripción y diferenciación de especies (Kong \& Iratchet 1995, Campello \& Bemvenuti 2002, Lloris \& Matallanas 2003, Díaz de Astarloa 2005, Dyer 2006, Matallanas \& Lloris 2006) y elaborar guías de identificación (Newsome 1977, Borodulina 1984, Hansel et al.1988; Gosztonyi \& Kuba 1996, Watt et al.1997).
En la laguna costera Mar Chiquita existen trabajos que describen aspectos del esqueleto de algunas especies de manera parcial (Díaz de Astarloa 1991), o integral (Díaz de Astarloa 1994, Segura \& Díaz de Astarloa 2004), pero en todos los casos son estudios puntuales no orientados a la comparación de los elementos óseos de las diferentes especies presentes en la citada laguna.

El objetivo del presente trabajo es realizar la descripción de once elementos óseos de las principales especies de peces de la laguna costera Mar Chiquita y confeccionar claves diagnósticas sobre la base de las diferencias más importantes encontradas entre las especies estudiadas, a saber: el clupeido Brevoortia aurea (Spix \& Agassiz, 1829), el mugílido Mugil platanus Günther 1880,el aterínido Odontesthes argentinensis (Valenciennes 1835), el paralíctido Paralichthys orbignyanus (Valenciennes, 1839), el pomatómido Pomatomus saltatrix (Linnaeus, 1758) y los esciénidos Micropogonias furnieri (Desmarest, 1823), Pogonias cromis (Linnaeus, 1766), Cynoscion guatucupa (Cuvier, 1830) y Menticirrhus americanus (Linnaeus, 1758).

\section{Material y métodos}

Se analizaron seis ejemplares de las nueve especies de peces mencionadas (tres de cada sexo), adultos y subadultos, provenientes de muestreos efectuados en la laguna costera Mar Chiquita durante los años 2005-2006. Los individuos se recolectaron con tres redes agalleras de monofilamento, de $25 \mathrm{~m}$ de longitud y $2 \mathrm{~m}$ de ancho, confeccionadas con mallero de diferentes tamaños: 57 , 68 y 120 mm. La identificación de las especies se basó sobre trabajos de Cousseau \& Perrotta (2004), Cousseau et al. (2001), Cousseau et al. (2004), Dyer (2006) y González-Castro (2007). Los rangos de longitud corporal de los peces (en longitud estándar) y las abreviaturas de las especies de peces utilizadas en las figuras del presente trabajo fueron: 265-300 mm para Brevoortia aurea $(\mathrm{Ba})$; 297-422 mm para Mugil platanus (Mp); 272-315 mm para Odontesthes argentinensis (Oa); 334-525 mm para Micropogonias furnieri (Mf); 342-407 mm para Pogonias cromis (Pc); 277-482 mm para Paralichthys orbignyanus (Po);178-250 mm para Pomatomus saltatrix (Ps); 427485 mm para Cynoscion guatucupa (Cg); y 227-293 mm para Menticirrhus americanus (Ma).

La desarticulación de los esqueletos fue realizada según la metodología propuesta por Díaz de Astarloa (1994). Para la nomenclatura de los huesos se siguió a Cervigón (1980) y a Rojo (1988). Se seleccionaron 11 elementos óseos del flanco izquierdo del cuerpo basándose en los criterios referentes a las características diagnósticas de los huesos y su grado de resistencia a la 
digestibilidad, aplicados por Gosztonyi \& Kuba (1996). Dichos elementos correspondieron a diversas regiones del cráneo y cintura pectoral: paraesfenoides, lacrimal, premaxilar, dentario, anguloarticular, palatino, hiomandibular, ceratohial, urohial, preopérculo y postemporal. Estos elementos fueron luego descritos y comparados morfológicamente entre las 9 especies de peces consideradas, y según las características diferenciales encontradas entre las especies se confeccionaron claves diagnósticas. Las observaciones se realizaron a simple vista y en casos particulares bajo lupa estereoscópica Kyowa SZM. Además, se registraron las dimensiones de los procesos óseos más notorios, mediante la utilización de un calibre digital MITUTOYO con precisión de $0,01 \mathrm{~mm}$. Se tomaron fotografías digitales de los huesos con una cámara Nikon COOLPIX L1.

\section{Resultados y discusión}

\section{Descripción osteológica}

\section{Paraesfenoides}

El paraesfenoides consta, anteriormente de una barra alargada y posteriormente de una superficie más expandida. Entre ambas partes del hueso se sitúan dos apófisis alares laterales (Fig. 1).
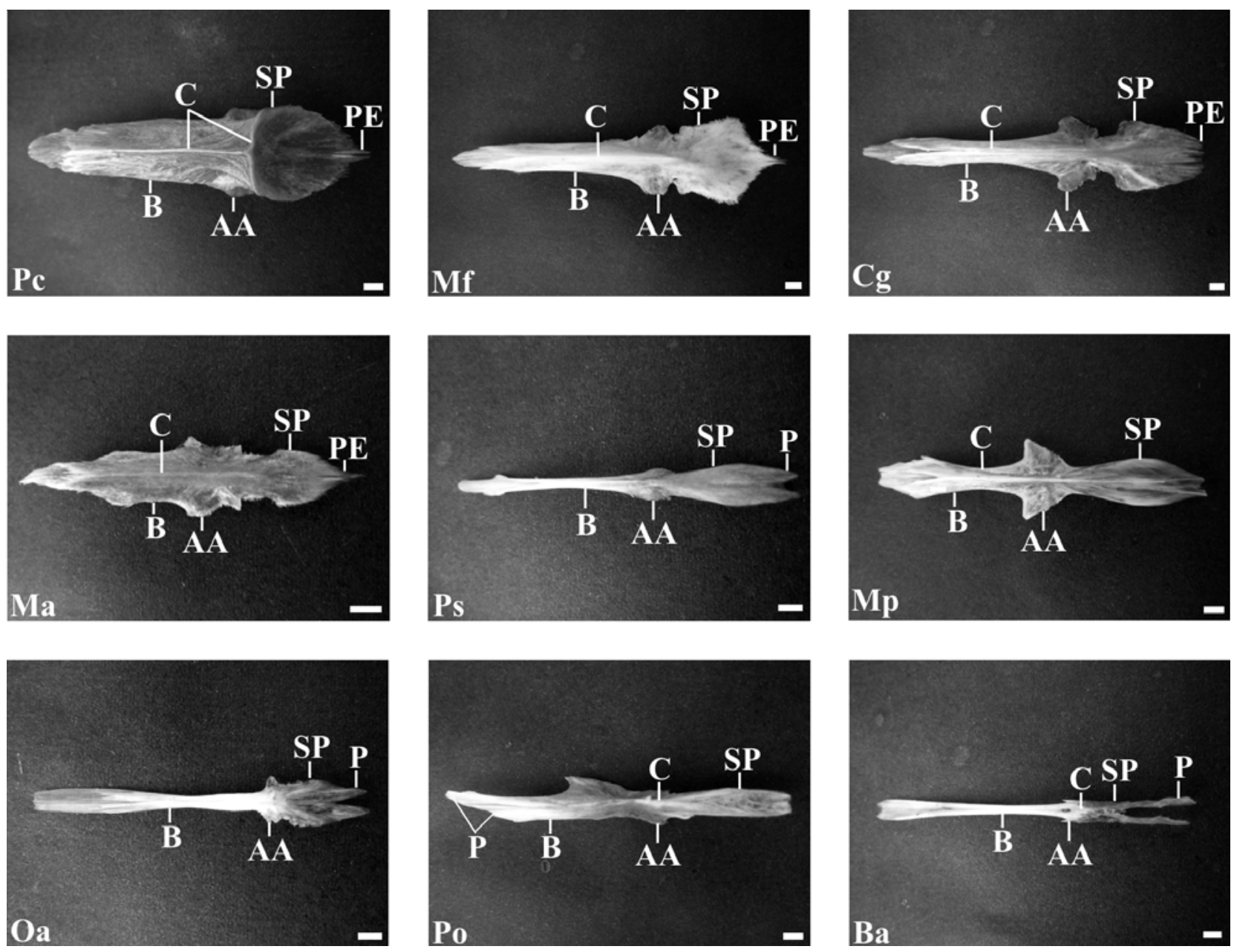

Figura 1

Fotografías del paraesfenoides en cara ventral para las 9 especies de peces analizadas. Abreviaturas: AA: apófisis alares; B: barra; C: cresta; P: proyecciones o procesos; PE: procesos espinosos; SP: superficie posterior. Escala: 3 mm

Photographies of the parasphenoid on ventral side for the 9 fish species analyzed. Abbreviations: AA: lateral wings; B: bar; C: crest; P: projections or processes; PE: thorny processes; SP: posterior surface. Scale bar: 3 mm 
La barra anterior es ancha en los esciénidos, $M$. platanus y $P$. orbignyanus y muy delgada en las demás especies examinadas. Presenta bordes laterales rectos en $P$. cromis; rectos hacia el extremo anterior, luego convexos y posteriormente cóncavos en $M$. americanus; anteriormente rectos y posteriormente cóncavos en $M$. platanus; asimétricos, siendo el izquierdo convexo y el derecho cóncavo, en $P$. orbignyanus; y ligeramente cóncavos en las demás especies. A lo largo de su superficie media se extiende una cresta sólo en los esciénidos analizados, $M$. platanus y $P$. orbignyanus. Su extremo anterior es aguzado y en punta en los esciénidos estudiados. En P. orbignyanus consta de dos proyecciones asimétricas, siendo la izquierda más corta. En M. platanus y en $B$. aurea, presenta dos pequeñas puntas. En $O$. argentinensis termina en un borde irregular y en $P$. saltatrix, en un borde redondeado.

Las apófisis alares son redondeadas en P. cromis, $M$. furnieri, C. guatucupa y P. saltatrix; presentan borde cóncavo en M. americanus; son irregulares, desiguales y presentan base ancha en $P$. orbignyanus; tienen forma triangular en $M$. platanus y en $O$. argentinensis; y presentan bordes rectos en $B$. aurea. Se posicionan aproximadamente hacia la mitad del hueso en $M$. platanus y $P$. orbignyanus, mientras que en el resto de las especies se ubican hacia el extremo posterior del hueso.

La superficie posterior, en los esciénidos estudiados, es ancha y presenta un borde posterior constituido por una serie de procesos espinosos, a través de los cuales se articula con el basioccipital. Los procesos espinosos son cortos en C. guatucupa y más largos constituyendo una punta en los demás esciénidos. Consta de bordes cóncavos en $M$. furnieri, convexos en P. cromis y M. americanus y aproximadamente rectos en C. guatucupa. Presenta una cresta transversal que la separa de la barra anterior, sólo en $P$. cromis. En el resto de las especies estudiadas, la superficie posterior es más delgada y no presenta la serie de procesos espinosos. Termina en un borde irregular en $P$. orbignyanus y M. platanus, en dos procesos largos y separados por una escotadura amplia en $B$. aurea y en dos proyecciones espinosas cortas, separadas por una pequeña escotadura en forma de $\mathrm{V}$ en $O$. argentinensis y $P$. saltatrix. Consta de bordes laterales aproximadamente rectos en $P$. orbignyanus y en $B$. aurea y redondeados en $M$. platanus, $O$. argentinensis y $P$. saltatrix. Presenta una cresta central paralela al eje del hueso sólo en $B$. aurea.

\section{Lacrimal}

El lacrimal tiene forma aproximadamente trapezoidal, con su extremo posteroventral alargado en los esciénidos (Fig. 2). Es triangular, con ambos extremos en punta, en $O$. argentinensis. En M. platanus también es triangular, pero su extremo anterior consta de dos procesos espinosos pequeños y el posterior es redondeado. En P. saltatrix y $B$. aurea es anteriormente ancho, de borde redondeado y posteriormente alargado, delgado y terminado en punta. En $P$. orbignyanus tiene forma de L invertida. Según Díaz de Astarloa (2005) el lacrimal izquierdo de P. orbignyanus difiere mucho del correspondiente al lado oculado, que presenta forma piramidal.

En los esciénidos el extremo posterior del lacrimal se divide en dos. El posteroventral es redondeado en $P$. cromis y $M$. americanus y termina en punta en $M$. furnieri y en C. guatucupa. El extremo posterodorsal es más corto que el ventral. Consta de base angosta en C. guatucupa y base ancha en las demás. Presenta bordes cóncavos en $P$. cromis, rectos en M. furnieri y C. guatucupa y convexos en $M$. americanus. El margen inferior del lacrimal es aserrado en $M$. americanus, $P$. saltatrix y M. platanus y liso en las otras especies estudiadas.

El lacrimal consta de un canal que protege la rama laterosensorial circumorbitaria. En los esciénidos el canal está interrumpido por tres puentes óseos que delimitan tres fenestras y una gran abertura en el margen superior. En P. cromis, los puentes óseos son más gruesos y las fenestras más pequeñas que en las demás especies. La abertura superior es circular en $P$. cromis, mientras que en los otros esciénidos presenta forma aproximadamente triangular.

En el resto de las especies estudiadas el canal no presenta puentes óseos. En P. orbignyanus se observa un surco somero que recorre el borde posterior de la rama superior y del vértice del hueso. En B. aurea y P. saltatrix el canal es poco notorio y muy delgado. Se extiende por el margen superior del hueso en $B$. aurea y por el inferior en $P$. saltatrix. En M. platanus se extiende a lo largo de la porción media del hueso y está delimitado por un pliegue óseo que se curva dorsalmente, destacándose en una saliente convexa. El lacrimal de $O$. argentinensis presenta un canal principal, en el borde superior, con aberturas en ambos extremos y tres canales secundarios que abren en el borde inferior, al igual que en O. mirinensis Bemvenuti, 1995 (Bemvenuti 1997). Sin embargo, en otros pejerreyes como O. smitti (Lahille, 1929), O. nigricans (Richardson, 1845) y O. perugiae (Evermann \& Kendall, 1906) hay sólo dos canales accesorios (Bemvenuti 1997, Piacentino 1999).

\section{Premaxilar}

El premaxilar puede presentar tres procesos: el ascendente, que permite que la boca sea protráctil (Rosen 1964, Alexander 1967), el articular y el maxilar (Figs. 3, 

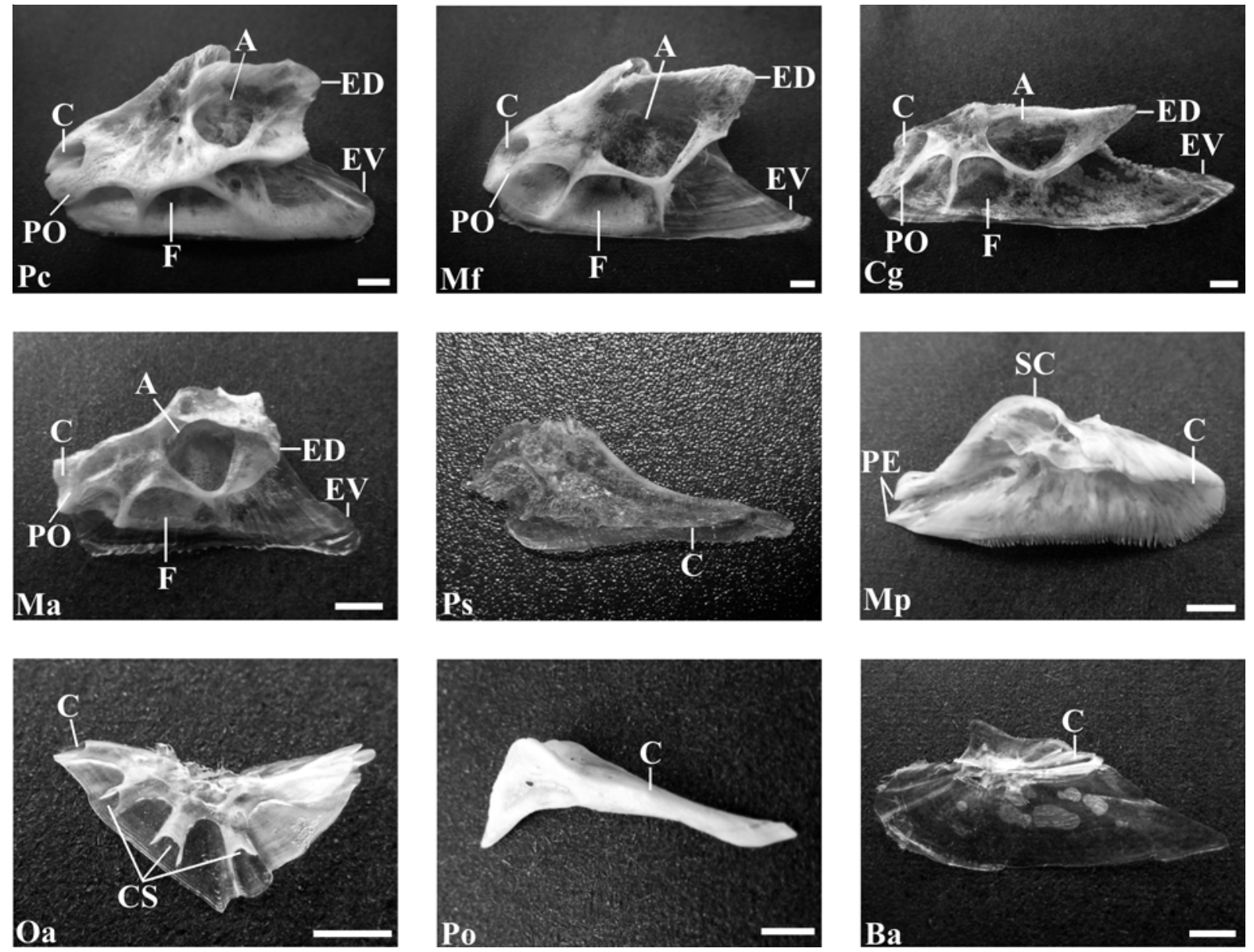

Figura 2

Fotografías del lacrimal en cara lateral externa para las 9 especies de peces analizadas. Abreviaturas: A: abertura; C: canal línea lateral; CS: canales secundarios; ED: extremo posterodorsal; EV: extremo posteroventral; F: fenestra; PE: procesos espinosos; PO: puente óseo; SC: saliente convexa. Escala: $\mathbf{3}$ mm

Photographies of the lachrymal in lateral outer side for the 9 fish species analyzed. Abbreviations: A: opening; C: laterosensory canal; CS: secondary canals; ED: posterodorsal end; EV: posteroventral end; F: fenestra; PE: thorny processes;

PO: osseous bridge; SC: convex salient. Scale bar: $3 \mathrm{~mm}$

4). En B. aurea el premaxilar está reducido y junto al maxilar constituye el margen superior de la boca (Segura \& Díaz de Astarloa 2004). Tiene forma triangular, con el extremo anterior en forma de $\mathrm{V}$ y el extremo posterior redondeado, sin procesos.

En $M$. platanus y O. argentinensis el premaxilar sólo consta de los procesos ascendente y maxilar, aunque para Rosen (1973) el patrón general en el premaxilar de los Acanthopterygii, es la presencia de apófisis articular. En M. platanus el proceso ascendente presenta forma rectangular, con un orificio central y se ubica en el extremo anterior del hueso. En O. argentinensis el proceso ascendente es largo, curvado anteriormente y se sitúa hacia la mitad del premaxilar. En M. platanus el proceso maxilar, de forma triangular, se encuentra en el extremo posterior del hueso. En $O$. argentinensis en cambio, presenta forma redondeada, expandiéndose dorsal y ventralmente a partir del extremo posterior del premaxilar.

En las otras especies estudiadas el premaxilar porta los tres procesos. El proceso ascendente, en todas las especies examinadas, se sitúa en el extremo anterior, es perpendicular al eje del hueso, delgado y termina en punta. La longitud de dicho proceso (medida desde su base sobre el eje horizontal del hueso hasta su extremo) es mayor a la longitud total del premaxilar (medida desde su extremo anterior al posterior) en $P$. cromis (media 121\%), es menor 

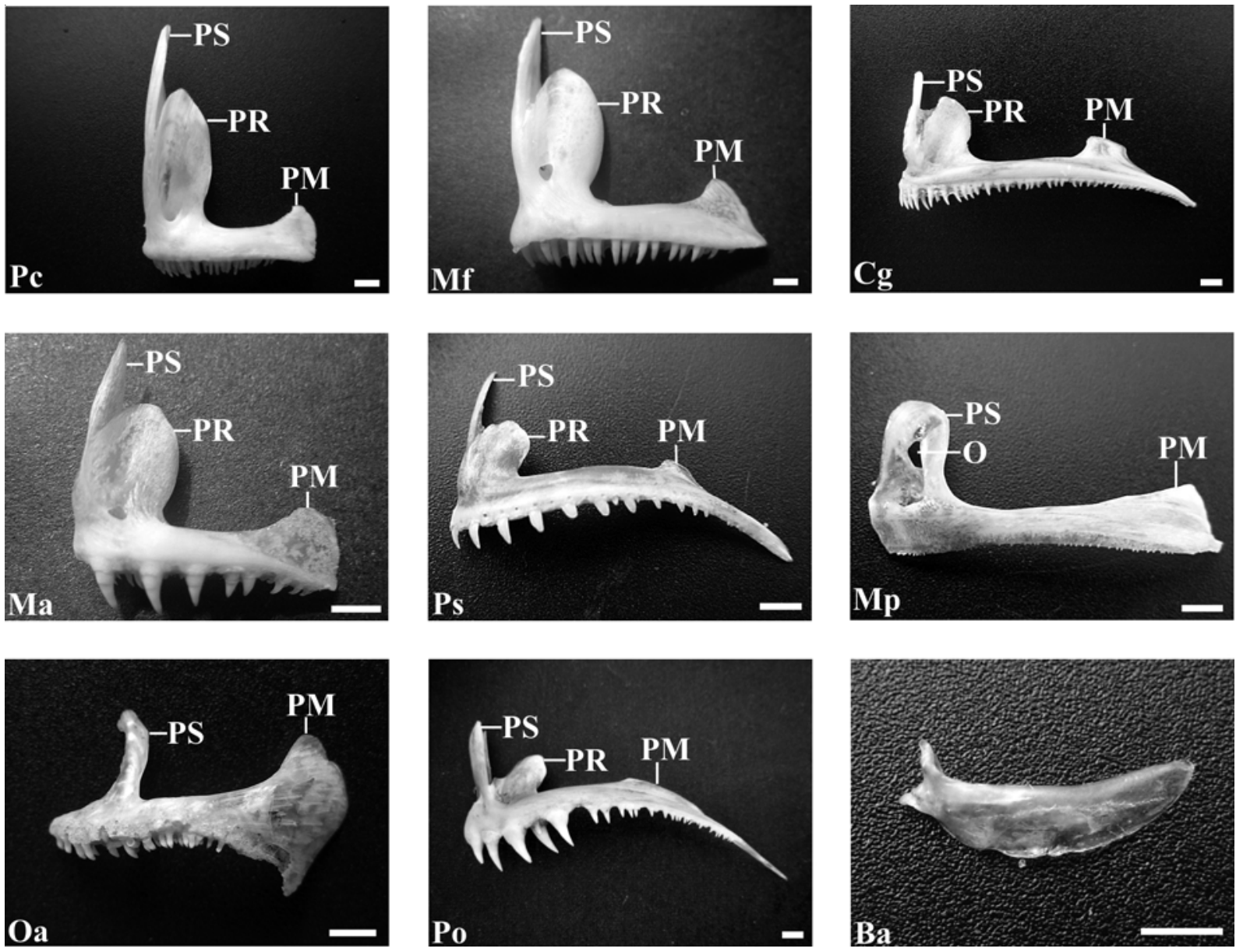

Figura 3

Fotografías del premaxilar en cara lateral externa para las 9 especies de peces analizadas. Abreviaturas: O: orificio; PM: proceso maxilar; PR: proceso articular; PS: proceso ascendente. Escala: $\mathbf{3}$ mm

Photographies of the premaxilla in lateral inner side for the 9 fish species analyzed. Abbreviations: O: orifice; PM; maxillar process; PR: articular process; PS: ascending process. Scale bar: $3 \mathrm{~mm}$

en $M$. furnieri y $M$. americanus (media 85 y 79\%, respectivamente) y es mucho menor en $P$. saltatrix, $C$. guatucupa y $P$. orbignyanus (media 43, 33, 30\%, respectivamente).

El proceso articular se ubica inmediatamente por detrás del ascendente y en todas las especies analizadas es oblicuo, ancho, de forma ovalada y más corto que el proceso ascendente. En las especies de esciénidos, la longitud del proceso articular (medida desde su base sobre el eje horizontal del hueso hasta su extremo) es aproximadamente 65-71\% de la longitud del ascendente, a diferencia de $P$. orbignyanus y $P$. saltatrix, que comprende alrededor de la mitad de la longitud del proceso ascendente (51 y 44\%, respectivamente).
Visto desde la cara externa (Fig. 3), el premaxilar de todas las especies analizadas exhibe los procesos ascendente y articular muy próximos entre sí, excepto en P. orbignyanus. Visto desde la cara interna (Fig. 4), ambos procesos se encuentran separados: (1) a través de una escotadura fina y profunda, que se inicia a la altura de la base del proceso articular, en $P$. cromis; (2) por una escotadura en forma de V, que se inicia más arriba de la base del proceso articular, en $M$. furnieri, $M$. americanus y P. saltatrix; (3) por una escotadura en forma de V, que se inicia a la altura de la base del proceso articular, en $C$. guatucupa.

El proceso maxilar se expande dorsalmente al eje del hueso: a nivel del extremo posterior en $P$. cromis, $M$. furnieri y $M$. americanus; de la mitad del hueso en $P$. orbignyanus; y del tercio posterior del hueso en $C$. 

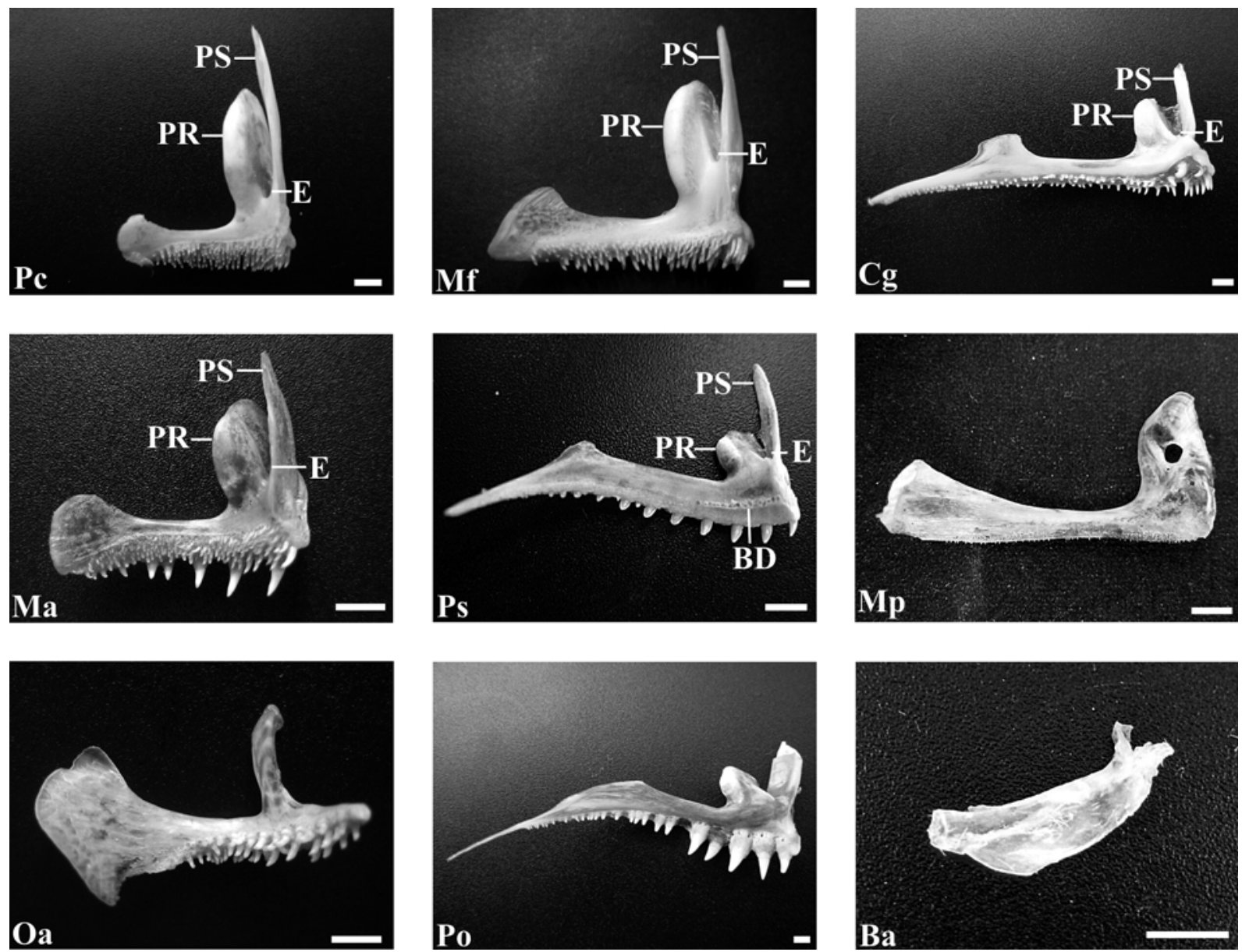

Figura 4

Fotografías del premaxilar en cara lateral interna para las 9 especies de peces analizadas. Abreviaturas: BD: banda de dientes; E: escotadura; PR: proceso articular; PS: proceso ascendente. Escala: $\mathbf{3}$ mm

Photographies of the premaxilla in lateral inner side for the 9 fish species analyzed. Abbreviations: BD: band of teeth; E: recess; PR: articular process; PS: ascending process. Scale bar: $3 \mathrm{~mm}$

guatucupa y $P$. saltatrix. Tiene forma triangular en $P$. cromis, $M$. furnieri y $M$. americanus y aproximadamente rectangular en las demás especies. En $P$. orbignyanus, $C$. guatucupa y $P$. saltatrix, el premaxilar termina posteriormente en una punta aguzada.

De todas las especies examinadas en este trabajo sólo el premaxilar de $B$. aurea es edéntulo. En las otras especies el hueso presenta dientes cuyo número va de 120 a 170 en $M$. furnieri, $P$. cromis, M. americanus y $C$. guatucupa, de 50 a 70 en $O$. argentinensis y M. platanus y de 23 a 36 en $P$. saltatrix y $P$. orbignyanus.

En P. cromis y M. furnieri, los dientes son viliformes, se disponen de manera desordenada y disminuyen de tamaño hacia el extremo posterior. En P. orbignyanus, son caniniformes, se organizan en una fila, siendo los anteriores muy grandes y los posteriores pequeños. En M. platanus, los dientes son unicúspides, muy pequeños, se disponen en una fila formando una banda y presentan el mismo tamaño. González Castro (2007) menciona que la mandíbula superior de $M$. platanus consta además de algunas hileras internas de dientes bicúspides, no encontrados en este trabajo, debido probablemente al método de extracción de los huesos con calor. En $M$. americanus los dientes del borde externo son cónicos, grandes, ordenados en una fila, mientras que los dientes interiores son viliformes, muy pequeños y distribuidos en varias filas. El resto de las especies analizadas presenta dientes cónicos. En C. guatucupa los dientes disminuyen de tamaño hacia el extremo posterior y se ubican anteriormente en tres filas y posteriormente en dos. En O. argentinensis son de tamaño similar y se encuentran 

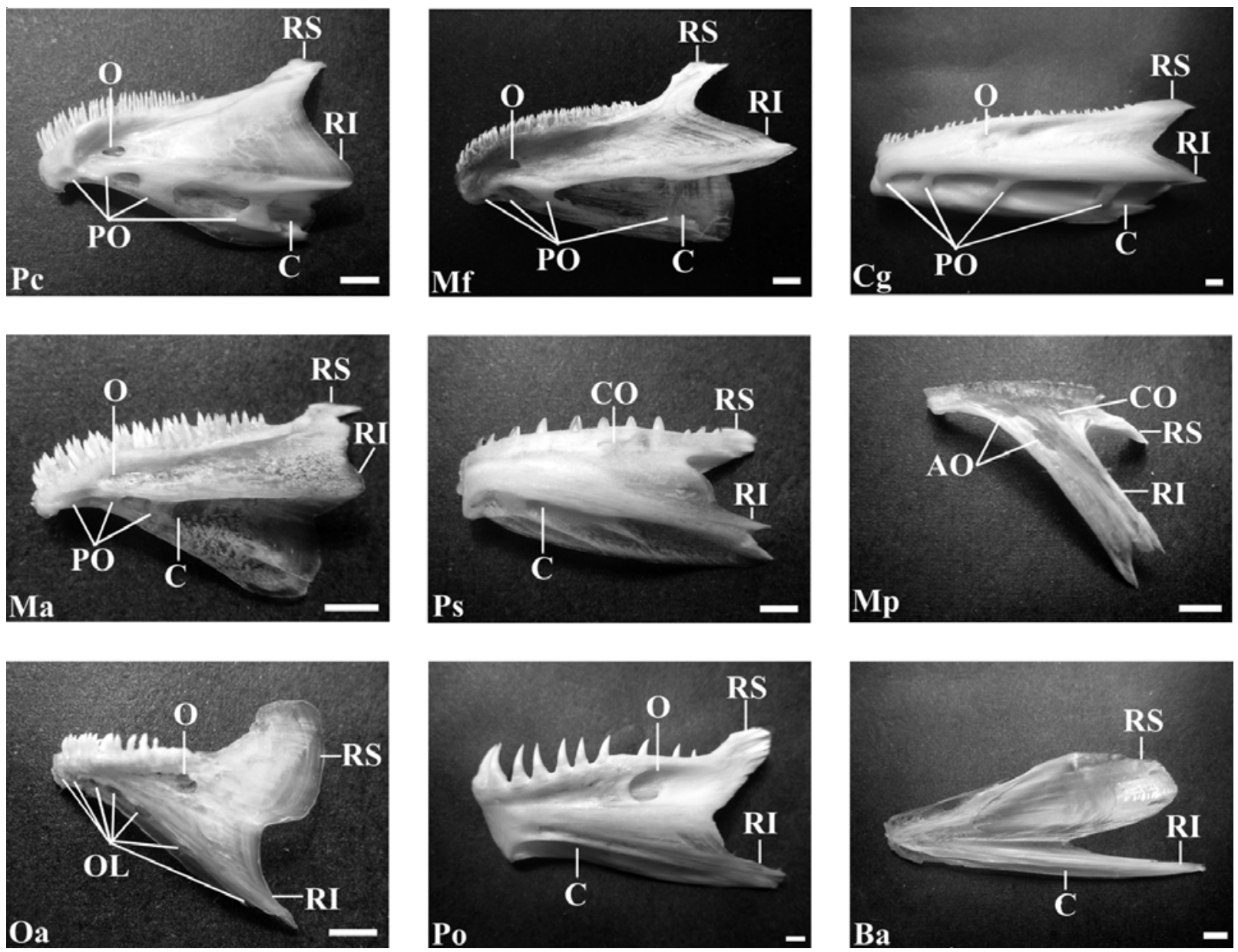

Figura 5

Fotografías del dentario en cara lateral externa para las 9 especies de peces analizadas. Abreviaturas: AO: aberturas ovaladas; C: canal línea lateral; CO: concavidad; O: orificio; OL: orificios laterales; PO: puentes óseos; RI: rama inferior; RS: rama superior. Escala: $3 \mathbf{~ m m}$

Photographies of the dentary in lateral outer side for the 9 fish species analyzed. Abbreviations: AO: oval openings; C: laterosensory canal; CO: concavity; O: orifice; OL: lateral orifices; PO: osseous bridge; RI: lower branch;

RS: upper branch. Scale bar: $3 \mathrm{~mm}$

dispuestos en dos filas. En P. saltatrix disminuyen de tamaño hacia el extremo posterior y están ordenados en una fila. En esta última especie el premaxilar exhibe, en la cara lateroventral interna, una banda de dientes muy pequeños, que se extiende hasta aproximadamente la mitad de la longitud del hueso.

\section{Dentario}

El dentario es aproximadamente triangular, con su borde posterior hendido en forma de horqueta constituyendo dos ramas, superior e inferior (Fig. 5).

La rama superior, en los esciénidos, es angosta y espinosa. En P. saltatrix, M. platanus y P. orbignyanus, también es angosta pero presenta un borde aproximadamente rectangular. En el resto de las especies analizadas la rama superior es muy ancha y de borde redondeado. La rama inferior termina posteriormente en borde redondeado en $P$. cromis y $M$. americanus, mientras que en $M$. furnieri y $C$. guatucupa termina en punta. En $P$. orbignyanus su extremo es irregular. En $P$. saltatrix y $M$. platanus termina en dos procesos espinosos separados por una escotadura en forma de V. En O. argentinensis y B. aurea es espinosa.

En la región lateroventral del dentario de los esciénidos se sitúa un surco interrumpido por puentes óseos, que aloja al canal mandibular de la línea lateral. Es profundo, recorre toda la longitud del hueso y presenta cuatro puentes óseos ubicados a lo largo del mismo, 
excepto en $M$. americanus, que exhibe tres puentes situados en la porción anterior del surco. En M. furnieri los tres primeros puentes óseos son anchos y el último es muy fino, mientras que en los otros esciénidos todos los puentes óseos son anchos. En el resto de las especies el canal no presenta puentes óseos. Es externo en $P$. saltatrix, $P$. orbignyanus y B. aurea, mientras que en $M$. platanus y $O$. argentinensis es interno. En $M$. platanus el canal interno abre a través de dos aberturas ovaladas en la parte superior de la rama. En $O$. argentinensis abre mediante siete orificios laterales, igual que en $O$. mirinensis y O. perugiae (Bemvenuti 1997), pero diferente de $O$. smitti y $O$. nigricans, que presentan cinco orificios (Piacentino 1999).

En la superficie laterodorsal, hacia el vértice anterior, se sitúa un gran orificio en $P$. cromis y $M$. furnieri, siendo muy pequeño en $M$. americanus. En $C$. guatucupa se encuentra un poro muy pequeño hacia la mitad del hueso. Hacia el extremo posterior del hueso se encuentra una gran abertura en $O$. argentinensis y $P$. orbignyanus y una pequeña concavidad en $P$. saltatrix y $M$. platanus.

B. aurea es la única de las especies estudiadas en este trabajo que presenta un dentario sin dientes. En las otras especies el hueso presenta dientes cuyo número va de 120 a 180 en $M$. furnieri, P. cromis y M. americanus, de 40 a 85 en C. guatucupa, O. argentinensis y M. platanus y de 10 a 16 en $P$. saltatrix y $P$. orbignyanus. En $P$. cromis, $M$. furnieri y $M$. americanus los dientes son viliformes, pequeños, ligeramente curvados hacia el interior de la boca y dispuestos en varias filas desordenadas. En $C$. guatucupa y $O$. argentinensis son cónicos, pequeños, se encuentran curvados hacia el interior de la boca y se disponen, anteriormente en tres filas y posteriormente en dos filas. En $P$. saltatrix son cónicos, grandes y se encuentran dispuestos en una fila, muy separados entre sí. En $P$. orbignyanus son caniniformes, muy grandes, curvados hacia el interior de la boca, disminuyen de tamaño hacia la parte posterior y se encuentran dispuestos en una fila. En M. platanus los dientes son muy finos y largos, del mismo tamaño, dispuestos en una fila formando una banda.

\section{Anguloarticular}

El anguloarticular es triangular y presenta tres grandes procesos: el coronoides, dirigido anterodorsalmente; el posterior, orientado hacia arriba, con una fosa de articulación para el cuadrado; y el proceso vertebral, de forma triangular, terminado en punta y dirigido hacia adelante. Además de los tres procesos anteriormente descriptos, el anguloarticular presenta una superficie ventral situada desde la mitad del hueso hacia atrás, provista de un proceso anterior más o menos desarrollado. En la superficie ventral se sitúa el canal sensorial de la línea lateral (Fig. 6).

La superficie ventral del anguloarticular en los esciénidos estudiados, es ancha y consta de un canal profundo que presenta un puente óseo. Las diferencias encontradas entre los esciénidos fueron las siguientes: (1) en C. guatucupa el proceso anterior es largo y de extremo aguzado, siendo más corto en los otros esciénidos estudiados; (2) el canal es ancho y de borde anterior recto en $M$. furnieri, mientras que en el resto de los esciénidos analizados es delgado y de borde anterior cóncavo; (3) en $P$. cromis el puente óseo es muy ancho y en los otros esciénidos es delgado. En las demás especies analizadas la superficie ventral es angosta y consta de un canal interno que abre dorsalmente al proceso anterior.

El proceso anterior es redondeado en $P$. saltatrix y espinoso en las otras especies. Es muy pequeño en $M$. platanus y $B$. aurea y más grande en las demás especies.

El proceso coronoides en $P$. cromis, $M$. furnieri, $C$. guatucupa, $M$. americanus, $P$. saltatrix y $M$. platanus, es bajo y termina anterodorsalmente en punta redondeada. Es alto, ancho y terminado en punta, en O. argentinensis y $P$. orbignyanus. En B. aurea es alto, delgado y termina en punta.

El proceso posterior es grande, termina en punta y se curva hacia arriba en las especies de la Familia Sciaenidae. En $O$. argentinensis y B. aurea es grande, curvado hacia arriba y de borde redondeado. En $P$. saltatrix es pequeño, curvado hacia arriba, redondeado y presenta un surco lateral continuación del canal interno. En M. platanus es muy pequeño, recto y termina en punta. En $P$. orbignyanus es grande, recto y termina hacia arriba en un proceso espinoso.

El retroarticular es un pequeño hueso de origen dérmico, unido firmemente al anguloarticular. Tiene forma de espina con la punta dirigida posteriormente y se sitúa en la base del puente óseo en $P$. cromis, $M$. furnieri y $M$. americanus. En C. guatucupa, $P$. saltatrix, M. platanus y $P$. orbignyanus, tiene forma triangular y se sitúa en el ángulo posteroinferior del hueso. En B. aurea el retroarticular es un hueso alargado en el eje horizontal y se encuentra unido a la parte posterior del anguloarticular (Segura \& Díaz de Astarloa 2004). El retroarticular de O. argentinensis no se ha podido separar en este trabajo, debido a que no se evidencia la sutura de articulación. Según Piacentino (1999) el retroarticular de O. smitti se articula al anguloarticular ventralmente por medio de una superficie acanalada, y dicha superficie también está presente en $O$. argentinensis. 

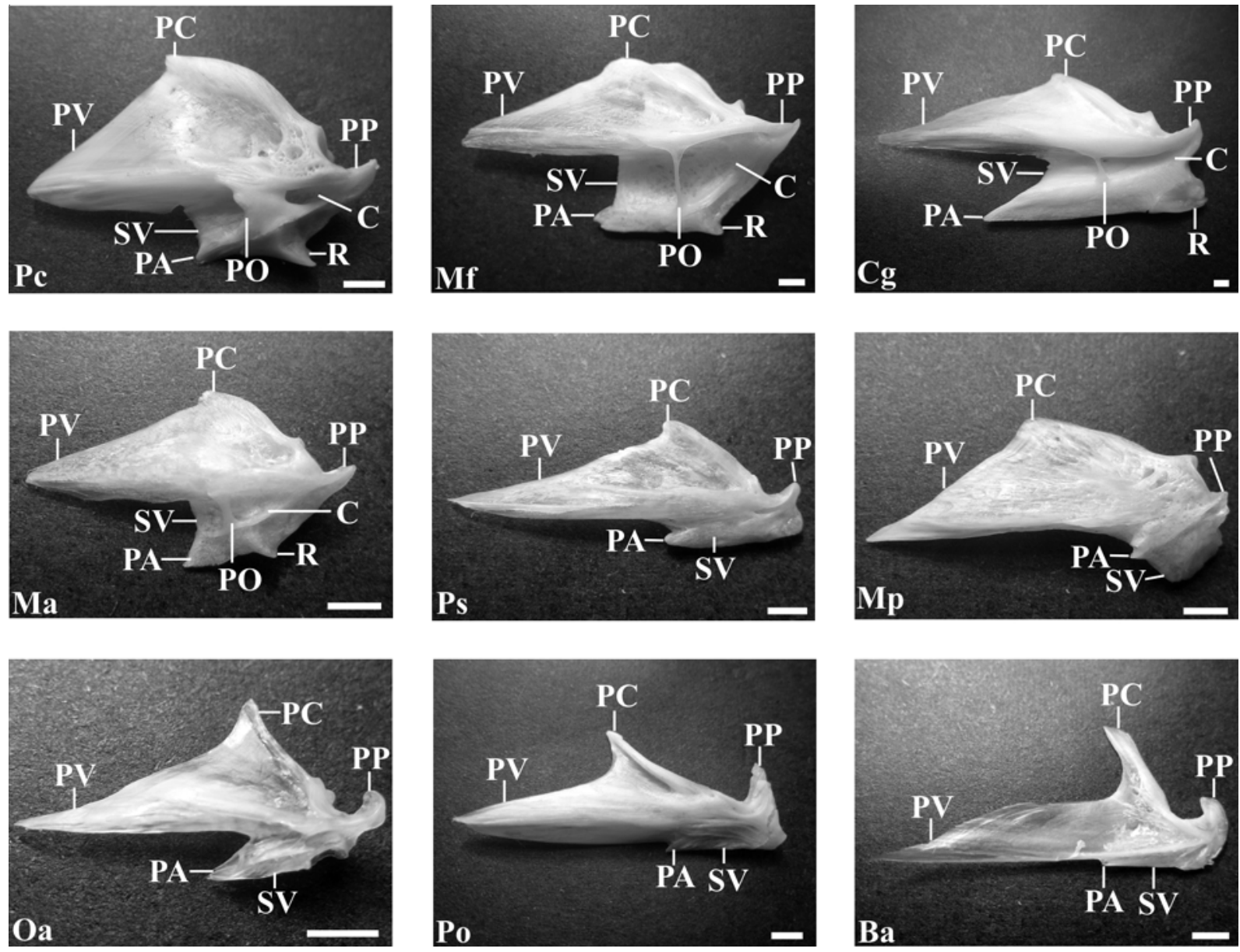

Figura 6

Fotografías del anguloarticular en cara lateral externa para las 9 especies de peces analizadas. Abreviaturas: C: canal línea lateral; PA: proceso anterior; PC: proceso coronoides; PO: puente óseo; PP: proceso posterior; PV: proceso ventral; R: retroarticular; SV: superficie ventral. Escala: $\mathbf{3} \mathbf{~ m m}$

Photographies of the anguloarticular in lateral outer side for the 9 fish species analyzed es. Abbreviations: C: laterosensory canal; PA: anterior process; PC: coronoid process; PO: osseous bridge; PP: posterior process;

$\mathrm{PV}$ : ventral process; R: retroarticular; SV: ventral surface. Scale bar: $3 \mathrm{~mm}$

\section{Palatino}

El palatino consta de dos superficies, ventral (oral) y dorsal (orbital), y de un proceso anterior (Fig. 7).

En las especies estudiadas pertenecientes a la Familia Sciaenidae presenta una fuerte cresta longitudinal que forma con la superficie ventral una gran concavidad, un cóndilo situado posterodorsalmente a la cresta para la articulación rostro-palatina que conecta al palatino con el neurocráneo (Jardim 1988), una saliente redondeada en el borde dorsal del proceso anterior y el extremo posterior terminado en punta. Las diferencias morfológicas encontradas entre los esciénidos, fueron las siguientes: (1) la cresta dorsal es recta en C. guatucupa, mientras que en el resto de los esciénidos se encuentra curvada; (2) el proceso anterior es corto y triangular en C. guatucupa y recto y largo en los otros esciénidos analizados; (3) entre el proceso anterior y la superficie ventral se encuentra una saliente convexa en $M$. furnieri y una pequeña escotadura en los demás esciénidos; (4) la superficie ventral presenta borde convexo en $C$. guatucupa, aproximadamente recto en $M$. americanus, anteriormente convexo y posteriormente cóncavo en $P$. cromis y anteriormente cóncavo y posteriormente convexo en $M$. furnieri; (5) el cóndilo dorsal es muy pequeño y triangular en C. guatucupa. En P. cromis y M. furnieri, termina dorsalmente en dos puntas separadas por una 

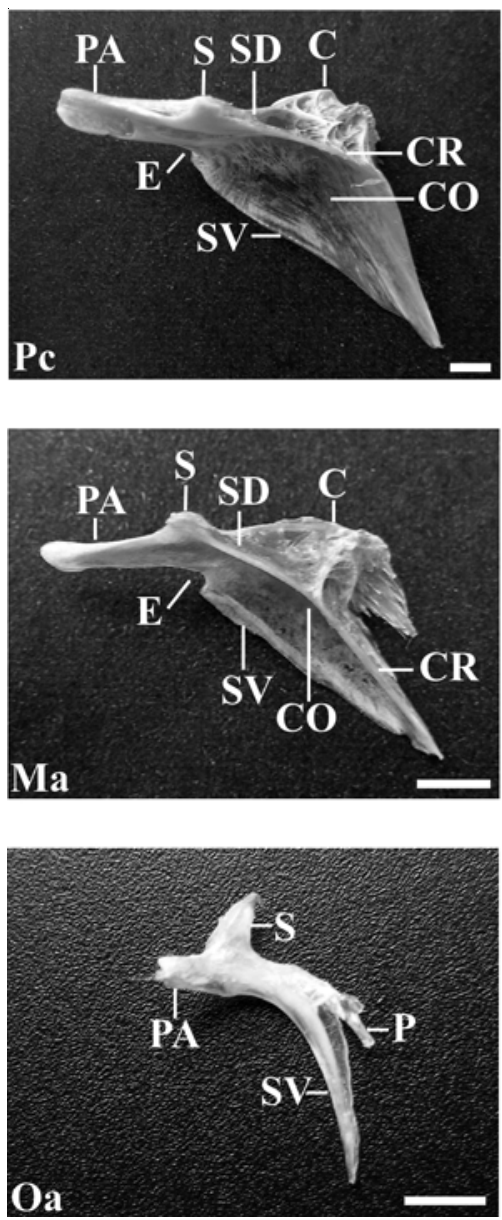
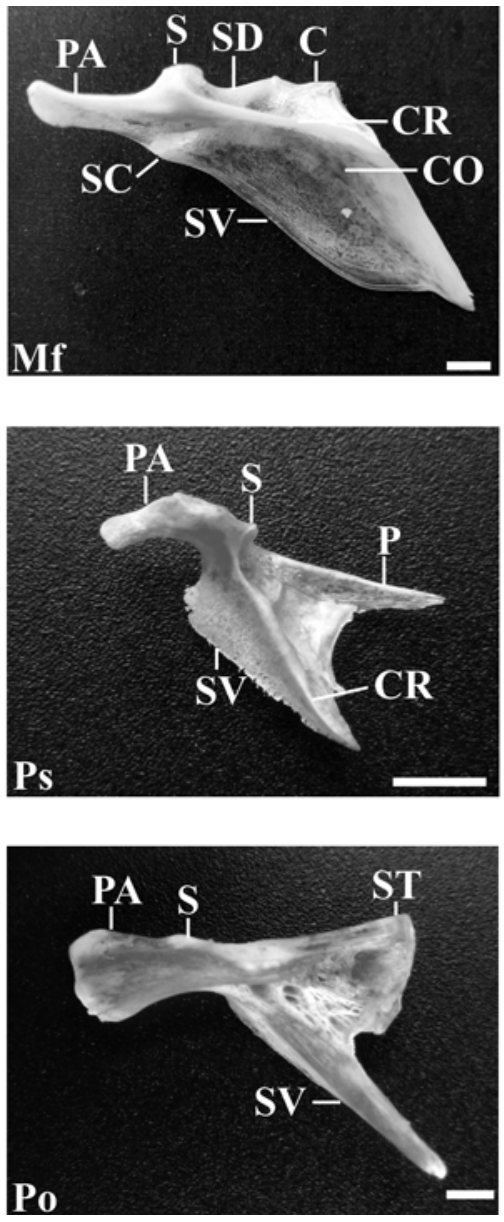

Figura 7
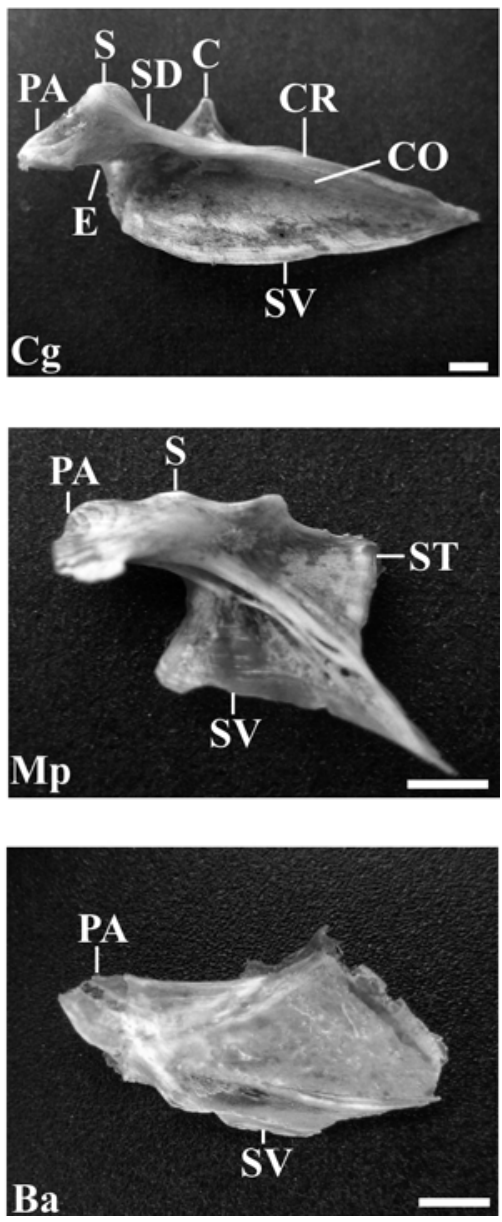

Fotografías del palatino en cara lateral externa para las 9 especies de peces analizadas. Abreviaturas: C: cóndilo; CO: concavidad; CR: cresta; E: escotadura; P: proceso; PA: proceso anterior; S: saliente; SC: saliente convexa; SD: superficie dorsal; ST: superficie triangular; SV: superficie ventral. Escala: $\mathbf{3} \mathbf{~ m m}$

Photographies of the in lateral outer side for the 9 fish species analyzed. Abbreviations: C: condyle; CO: concavity; CR: crest; E: recess; P: process; S: salient; SC: convex salient; SD: dorsal surface; ST: triangular surface; SV: ventral surface. Scale bar: $3 \mathrm{~mm}$

concavidad. En M. americanus termina en un borde redondeado y presenta una saliente laminar posterior.

En las otras especies estudiadas, la cresta está ausente, excepto en $P$. saltatrix, que presenta una cresta baja y recta que no forma una concavidad con la superficie ventral.

La saliente ubicada en el borde superior del proceso anterior está ausente en $B$. aurea; es redondeada y muy baja en $M$. platanus y $P$. orbignyanus; y es triangular en $P$. saltatrix y $O$. argentinensis, siendo muy grande en este último.

Del borde superior, hacia la mitad del hueso, se proyecta un proceso muy largo y aguzado en $P$. saltatrix y corto y en forma de varilla en $O$. argentinensis. En $M$. platanus y $P$. orbignyanus se proyecta dorsalmente una superficie de forma aproximadamente triangular.

El extremo posterior del hueso es espinoso en la mayoría de las especies analizadas, excepto en $B$. aurea, que presenta un palatino ancho y de forma triangular.

El proceso anterior es largo, delgado y curvado ventralmente en $P$. saltatrix; largo, ancho y redondeado en $M$. platanus y $P$. orbignyanus; corto y delgado en $O$. argentinensis; y redondeado, ancho y poco expandido en $B$. aurea. 
La superficie ventral consta de un borde cóncavo en $O$. argentinensis, triangular en $M$. platanus, recto en $P$. saltatrix y $P$. orbignyanus y convexo en $B$. aurea. En $P$. saltatrix la superficie ventral presenta numerosos dientes diminutos.

\section{Hiomandibular}

El hiomandibular presenta, en su región dorsal, dos apófisis: la primera, denominada apófisis superior o auricular, está provista de dos cóndilos articulares (anterodorsal y posterodorsal) y la segunda, denominada apófisis opercular, está dirigida hacia atrás y provista del cóndilo posterior. También, consta de una expansión anterior y de una cresta que se extiende lateralmente, sobre el eje vertical (Fig. 8).

Las especies de la Familia Sciaenidae presentan un hiomandibular que consta de una proyección espinosa que se extiende dorsalmente con respecto al cóndilo posterodorsal, de una expansión anterior fuertemente osificada, con borde externo irregular y de una cresta longitudinal que se bifurca en dos ramas abiertas, una hacia el borde anterior y otra por el borde posterior, delimitando canales o concavidades amplias. Las diferencias observadas en los esciénidos están referidas a: (1) la proyección espinosa es gruesa y está relacionada con la apófisis opercular a través de una lámina muy osificada, de borde aproximadamente recto en $P$. cromis. En el resto de las especies es delgada. Se relaciona con la apófisis opercular a través de una lámina de borde aproximadamente recto en C. guatucupa y de borde convexo en $M$. furnieri y M. americanus; (2) la expansión anterior presenta un borde externo aproximadamente redondeado en $P$. cromis, triangular en C. guatucupa y rectangular en $M$. furnieri y $M$. americanus; (3) en $P$. cromis y C. guatucupa la cresta sobresale del hueso posteriormente y se bifurca en dos ramas paralelas, generando un canal profundo. En $M$. furnieri y $M$. americanus la cresta sobresale del hueso anteriormente. En $M$. furnieri se bifurca inferiormente en dos ramas, una se curva anteriormente y la otra sigue paralela al eje, generando una gran concavidad dividida por un tabique óseo. En $M$. americanus, se divide en dos ramas muy abiertas, la anterior es perpendicular al eje y la posterior es paralela, delimitando una concavidad poco profunda; (4) el ancho máximo del hiomandibular (medido desde el extremo del cóndilo anterodorsal al borde exterior del cóndilo posterior), en relación a la longitud total del hueso (medida desde el extremo ventral al borde dorsal del cóndilo posterodorsal) es mucho menor en P. cromis (46$51 \%$ ) que en los otros esciénidos estudiados (76-79\% en M. furnieri, $74-80 \%$ en C. guatucupa y 79-87\% en $M$. americanus).
En $M$. platanus, sobre el cóndilo posterodorsal del hiomandibular, surge una proyección lateral espinosa, ausente en el resto de las especies. Además, presenta cóndilos muy prominentes y gruesos. El hiomandibular de $B$. aurea consta de una apófisis auricular plana, con una profunda hendidura en forma de V. En P. saltatrix el cóndilo anterodorsal es más bien anterior, encontrándose en el mismo eje que el cóndilo posterior. En $P$. orbignyanus el cóndilo posterior se encuentra inclinado ventralmente.

La expansión anterior se encuentra unida al eje vertical hasta la tercera parte del hueso en P. saltatrix, O. argentinensis y $P$. orbignyanus, hasta la mitad del hueso en $M$. platanus y hasta la primera parte del hueso en $B$. aurea. En $P$. saltatrix, la expansión anterior tiene borde redondeado. En $M$. platanus presenta un borde festoneado. En O. argentinensis exhibe una forma aproximadamente rectangular y borde irregular. En $P$. orbignyanus presenta forma triangular. En B. aurea, la expansión anterior se curva anteriormente terminando en un apéndice espinoso dirigido hacia arriba.

La cresta es ancha hacia el extremo superior y se adelgaza hacia la parte posterior del eje en $P$. saltatrix y en B. aurea. En P. saltatrix, cubre al cóndilo posterodorsal y hacia el extremo inferior del eje se bifurca en dos pequeñas ramas que delimitan un canal poco profundo. En $B$. aurea presenta un gran orificio aproximadamente ovalado y surcos en los bordes anterior y posterior. En $M$. platanus y $P$. orbignyanus se inicia sobre la porción anterior de la apófisis auricular. En M. platanus sobresale anteriormente del eje en una punta y luego se continúa hacia el final del eje por el borde posterior. En $P$. orbignyanus comienza y finaliza en un proceso espinoso y delimita un surco en el borde anterior. En $O$. argentinensis se inicia en la base de la apófisis opercular, sobresale posteriormente y hacia el extremo inferior del eje vertical presenta dos pequeños orificios, uno lateral en el borde posterior y otro en la cara externa. Estos poros permiten el paso de las ramificaciones hioidea y mandibular respectivamente y de la ramificación hiomandibular del nervio facial VII (Bemvenuti 1995).

El hiomandibular de $P$. orbignyanus y $B$. aurea es comparativamente más largo que el del resto de las especies estudiadas. El ancho máximo en relación a la longitud total del hueso es $39-44 \%$ en $P$. orbignyanus, 40-46\% en B. aurea, 49-54\% en $M$. platanus, 58-65\% en $P$. saltatrix y 64-67\% en O. argentinensis. En B. aurea, el eje vertical del hiomandibular es cilíndrico y delgado, mientras que en $P$. orbignyanus es aplanado y ancho. 

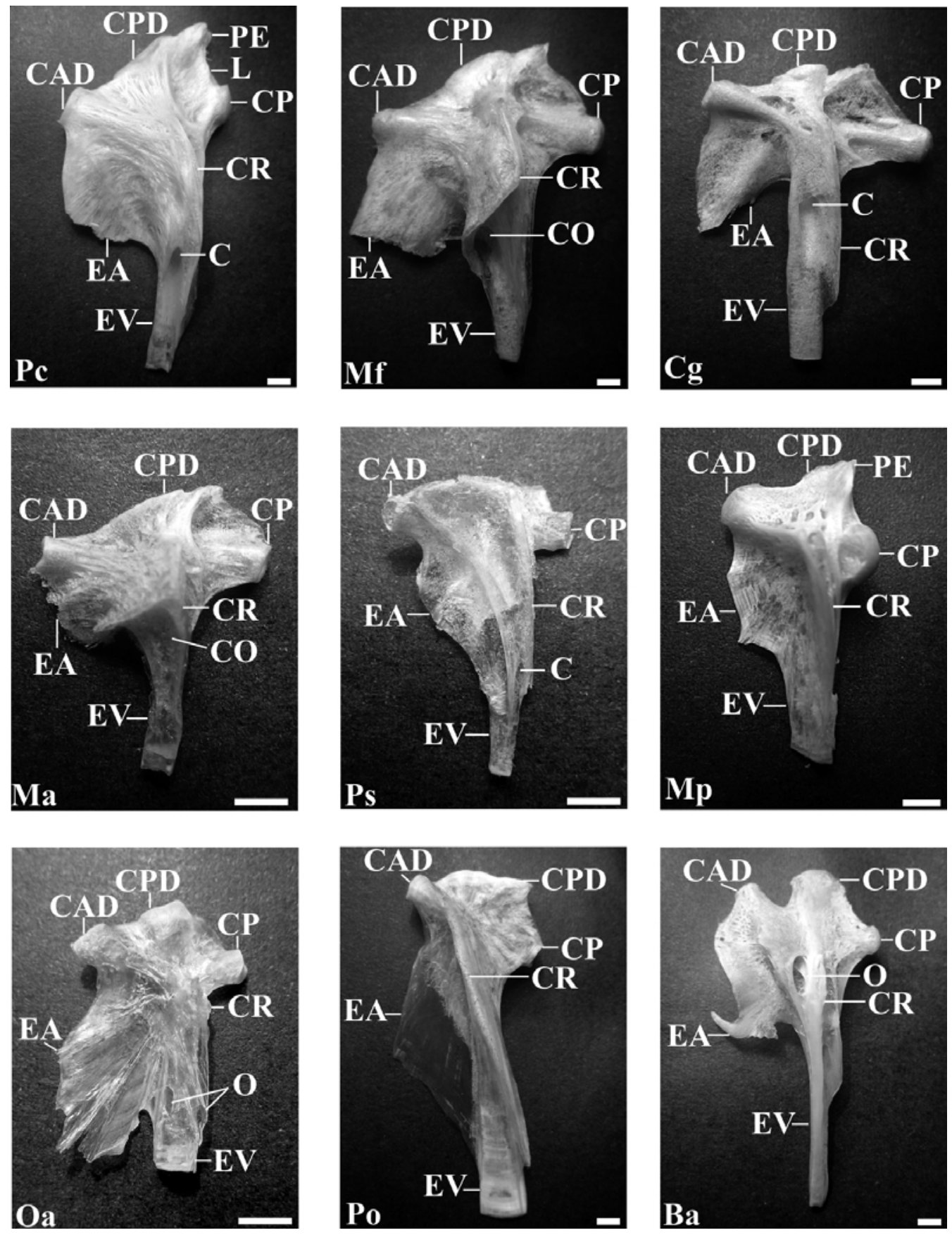

Figura 8

Fotografías del hiomandibular en cara lateral externa para las 9 especies de peces analizadas. Abreviaturas: C: canal; CAD: cóndilo anterodorsal; CO: concavidad; CP: cóndilo posterior; CPD: cóndilo posterodorsal; CR: cresta; EA: expansión anterior; EV: eje vertical; L: lámina; O: orificio; PE: proyección espinosa. Escala: 3 mm

Photographies of the hyomandibular in lateral outer side for the 9 fish species analyzed. Abbreviations: C: channel; CAD: anterodorsal condyle; CO: concavity; CP: posterior condyle; CPD: posterodorsal condyle; CR: crest; EA: anterior expansion; EV: vertical axis; L: lamina; O: orifice; PE: thorny projection. Scale bar: $3 \mathrm{~mm}$ 

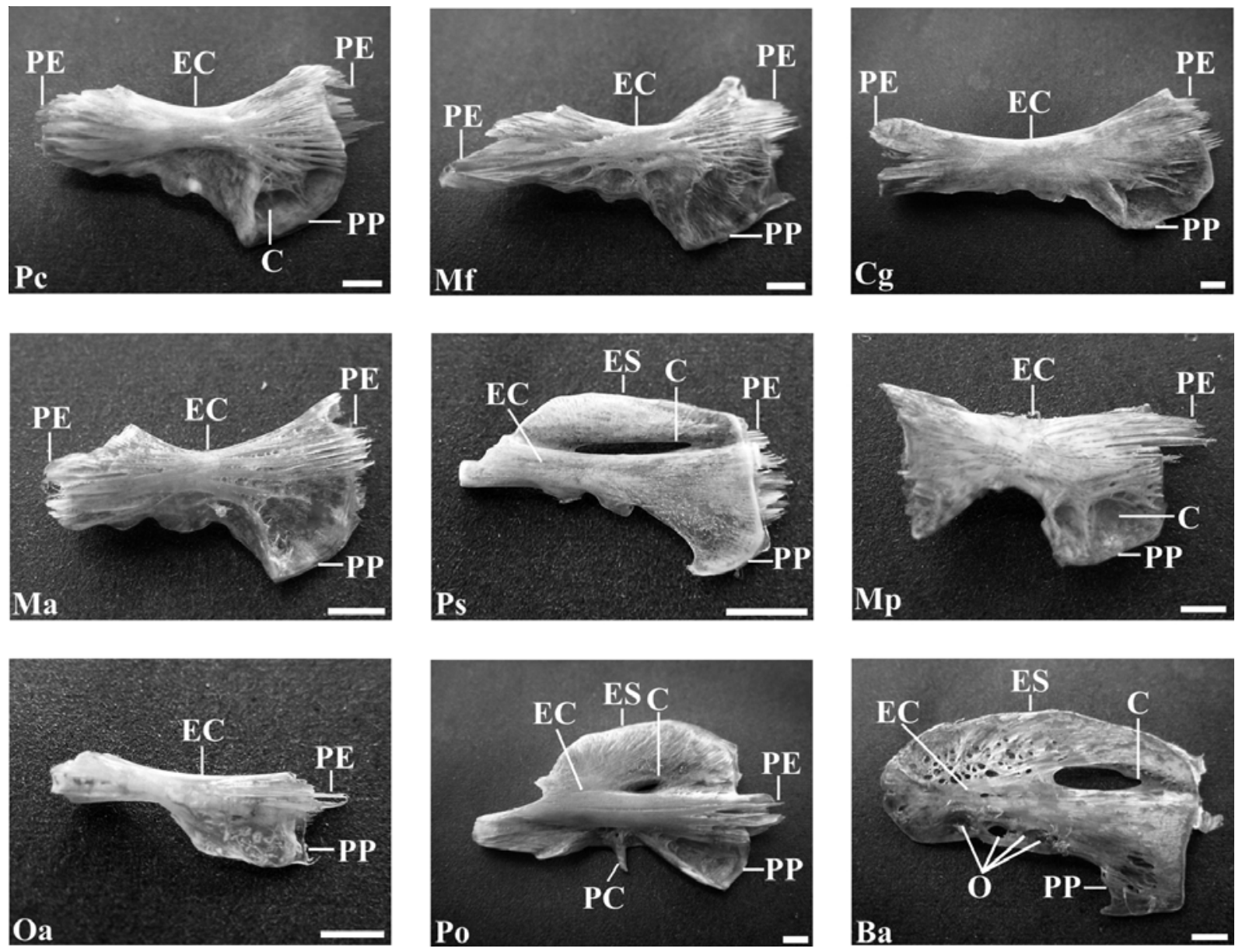

Figura 9

Fotografías del ceratohial en cara lateral externa para las 9 especies de peces analizadas. Abreviaturas: C: concavidad; EC: eje central; ES: expansión semicircular; O: orificios; PC: proceso central; PE: procesos espinosos; PP: porción posteroinferior. Escala: $3 \mathrm{~mm}$

Photographies of the ceratohyal in lateral outer side for the different studied species. Abbreviations: C: concavity; EC: central axis; ES: semicircular expansion; O: orifices; PC: central process; PE: thorny processes; PP: posteroinferior portion. Scale bar: $3 \mathrm{~mm}$

\section{Ceratohial}

El ceratohial es un hueso alargado con su porción posterior expandida (Fig. 9). En la mayoría de las especies examinadas, el ceratohial se vincula con el epihial mediante una delgada lámina de cartílago y una serie de procesos espinosos que interdigitan con sendos procesos provenientes del epihial, a excepción de $B$. aurea, en la que el ceratohial no presenta procesos espinosos.

El extremo anterior del ceratohial, en los esciénidos estudiados, está constituido por proyecciones espinosas y se encuentra ligeramente expandido en relación al eje central del hueso. En $M$. furnieri presenta una proyección inferior más larga, a diferencia del resto de los esciénidos.
En las demás especies el extremo anterior no presenta proyecciones espinosas. Presenta borde irregular en $M$. platanus y $O$. argentinensis, redondeado en $B$. aurea, mientras que en las demás especies analizadas tiene forma de varilla: corta y cilíndrica en $P$. saltatrix y larga y aplanada en $P$. orbignyanus. En O. argentinensis está ligeramente expandido con respecto al eje central del hueso, mientras que en $M$. platanus se encuentra muy expandido. En B. aurea es ancho, pero no se encuentra más expandido que el eje central del hueso. En las demás especies es muy delgado.

El eje central del hueso se encuentra comprimido y presenta un borde superior cóncavo en los esciénidos, $M$. platanus y $O$. argentinensis. En las demás especies 
analizadas el eje del hueso presenta una expansión superior de forma semicircular y de borde convexo. En la base de dicha expansión se sitúa una concavidad ovalada en $B$. aurea, una fosa alargada en $P$. saltatrix y un pequeño orificio en $P$. orbignyanus, que en este último probablemente constituye el paso de la arteria hioidea (Rojo 1988).

El borde inferior del ceratohial es ondulado en las especies de la Familia Sciaenidae y en P. saltatrix. En $M$. platanus y O. argentinensis es cóncavo, mientras que en B. aurea es irregular y presenta cuatro orificios pequeños. En $P$. orbignyanus, en cambio, consta de dos concavidades separadas por un pequeño proceso central.

El extremo posterior del ceratohial de las especies de la Familia Sciaenidae, se encuentra expandido superior e inferiormente, presenta borde convexo y procesos espinosos, para la articulación con el epihial. Las diferencias morfológicas encontradas con respecto al extremo posterior del ceratohial, entre los esciénidos, fueron: (1) en $P$. cromis, los procesos espinosos se cortan en el borde posterior de la cara externa; (2) la porción posteroinferior externa exhibe una serie de concavidades, que en $P$. cromis son muy notorias y definidas, mientras que en las otras especies son muy someras; (3) el extremo posterior termina inferiormente en punta, excepto en $C$. guatucupa en la que termina en un borde redondeado.

En las demás especies, el extremo posterior del ceratohial se encuentra expandido inferiormente. La porción posteroinferior exhibe una forma aproximadamente rectangular en $O$. argentinensis, triangular en $P$. orbignyanus y cuadrangular en las otras especies. En M. platanus, la porción posteroinferior externa presenta una serie de concavidades someras. En $P$. saltatrix y $B$. aurea, se curva hacia adelante en un pequeño proceso espinoso.

\section{Urohial}

En la mayoría de las especies analizadas el urohial tiene forma aproximadamente triangular y consta, anteriormente, de una cabeza tripartita compuesta por dos apófisis ventrolaterales y una apófisis impar y, posteriormente, de dos crestas laterales que delimitan una lámina dorsal y una ventral (Fig. 10).

En $P$. orbignyanus, el urohial tiene forma de 'boomerang', con vértice dirigido hacia atrás y dos brazos anteriores, sin cabeza tripartita ni crestas. En B. aurea es alargado, comprimido dorsoventralmente y curvado hacia arriba.

La apófisis impar se proyecta hacia adelante en $C$. guatucupa y B. aurea y se encuentra en posición dorsal en el resto de las especies analizadas.

Las crestas se extienden a lo largo del hueso, excepto en $B$. aurea, en la que se inician hacia la mitad posterior del hueso.

La lámina dorsal es muy angosta en C. guatucupa, $O$. argentinensis y $B$. aurea, mientras que en el resto de los esciénidos, $P$. saltatrix y $M$. platanus es más amplia. Presenta un borde externo convexo en los esciénidos y $M$. platanus, cóncavo en $B$. aurea, y aproximadamente recto en $O$. argentinensis y $P$. saltatrix. En esta última especie, la lámina dorsal y la apófisis impar se encuentran unidas.

La lámina ventral se encuentra ausente en M. platanus. En $B$. aurea es muy angosta, mientras que en las otras especies examinadas es más amplia. El borde inferior de la lámina ventral es convexo en todas las especies analizadas, excepto en $O$. argentinensis, en el que es recto y se expande posteriormente en dos prolongaciones laterales en forma de pequeñas alas. El borde posterior de la lámina ventral es cóncavo en $P$. cromis y $M$. furnieri; convexo en $M$. americanus y $B$. aurea; recto en $P$. saltatrix y ondulado en C. guatucupa y O. argentinensis.

El extremo posterosuperior del hueso se bifurca en proyecciones espinosas laterales en O. argentinensis. En $M$. platanus es convexo, mientras que en las demás especies analizadas el hueso termina posteriormente en punta, siendo ésta muy larga en $P$. cromis. El extremo posteroinferior del hueso se bifurca en salientes espinosas en $M$. platanus. En $M$. americanus es convexo, mientras que en las demás especies estudiadas es espinoso.

\section{Preopérculo}

El preopérculo presenta forma de media luna o de L con la curvatura hacia delante y presenta dos ramas, una superior y otra inferior (Fig. 11). En todas las especies estudiadas la rama superior del preopérculo es más larga que la inferior, excepto en $O$. argentinensis, en el cual ambas ramas presentan similar longitud.

En $M$. furnieri el borde posterior del preopérculo presenta varias proyecciones espinosas pequeñas y dos espinas muy desarrolladas. En C. guatucupa, $M$. americanus y $P$. saltatrix es aserrado. En el resto de las especies el borde posterior del preopérculo es liso. En $B$. aurea es particular la presencia de una pequeña escotadura a continuación del extremo posterior en punta del hueso.

El preopérculo es atravesado longitudinalmente por un canal o surco, donde se aloja la rama preopercular del canal sensorial. En los esciénidos, el canal se encuentra parcialmente abierto, ya que presenta una serie de puentes 

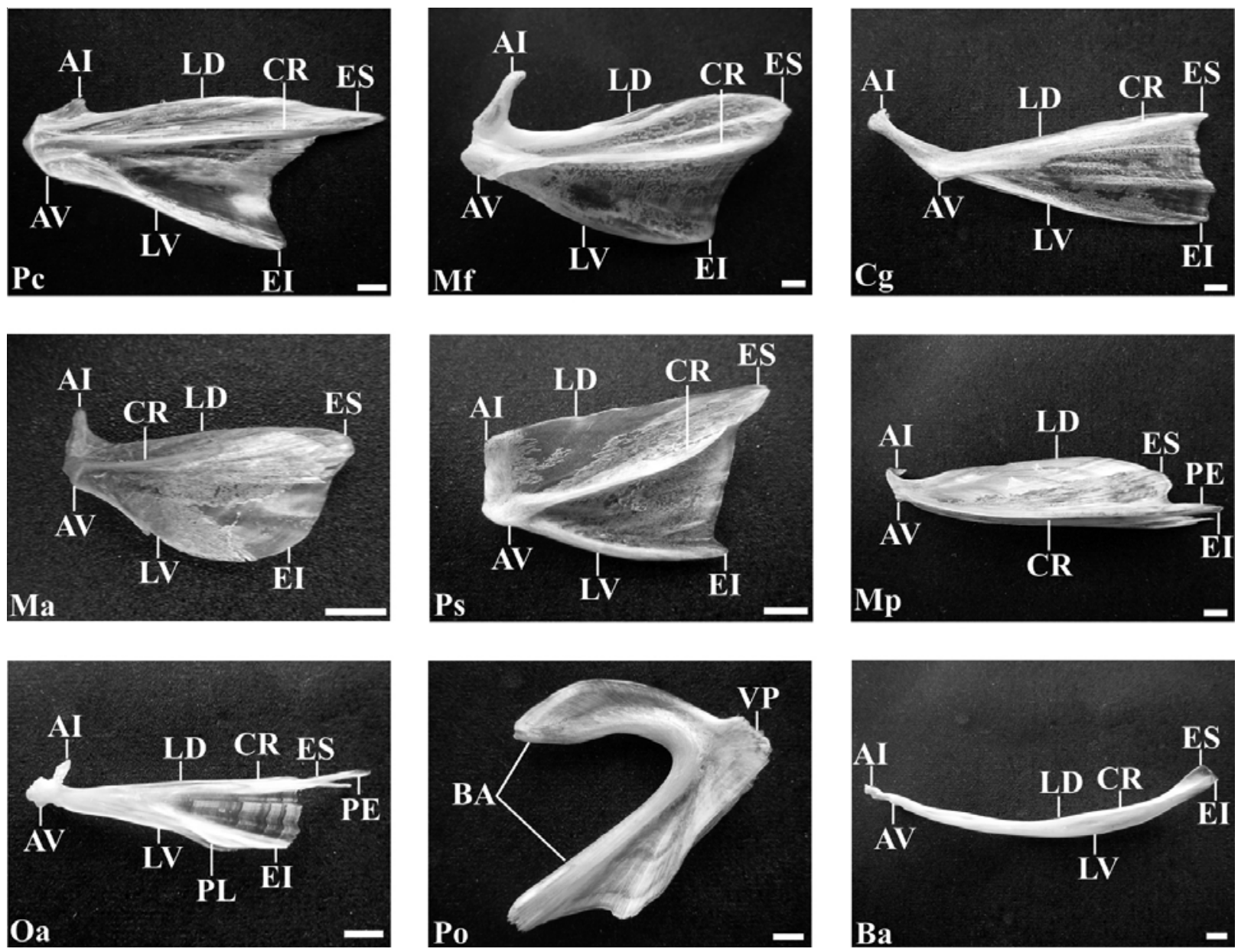

Figura 10

Fotografías del urohial en cara lateral externa para las 9 especies de peces analizadas. Abreviaturas: AI: apófisis impar; AV: apófisis ventrolaterales; BA: brazos anteriores; CR: cresta; EI: extremo posteroinferior; ES: extremo posterosuperior; LD: lámina dorsal; LV: lámina ventral; PE: procesos espinosos; PL: prolongaciones laterales; VP: vértice posterior. Escala: 3 mm

Photographies of the urohyal in lateral outer side for the 9 fish species analyzed. Abbreviations: AI: unpaired apophysis; AV: ventrolateral apophysis; BA: anterior arms; C: crest; EI: posteroinferior end; ES: posterosuperior end; LD: dorsal lamina;

LV: ventral lamina; PE: thorny processes; PL: lateral extensions; VP: posterior vertex. Scale bar: $3 \mathrm{~mm}$

óseos. Los puentes óseos son anchos en P. cromis y delgados en las otras especies. En C. guatucupa, terminan lejos del borde posterior. Delimitan concavidades cuadrangulares en C. guatucupa y M. americanus y semicirculares en $P$. cromis y $M$. furnieri.

En las demás especies analizadas no se observan puentes óseos. En B. aurea, el surco se encuentra abierto, es angosto y en su región ventral se ramifica en pequeños canales radiales. En $P$. orbignyanus el canal es cerrado, sólo abre al exterior a través de un poro en ambos extremos del hueso. El canal del preopérculo puede encontrarse: (1) abierto a lo largo de la rama superior y cerrado a lo largo de la rama inferior en M. platanus; (2) parcialmente abierto a lo largo de la rama inferior y cerrado a lo largo de la rama superior en P. saltatrix; (3) parcialmente abierto a lo largo de ambas ramas en $O$. argentinensis. Las especies de Odontesthes estudiadas por Bemvenuti (1997) también presentaron preopérculos con canales parcialmente abiertos, pero a diferencia de $O$. argentinensis, $O$. mirinensis consta de un canal totalmente abierto a lo largo de la rama superior y $O$. perugiae de un canal abierto a lo largo de la rama inferior. 

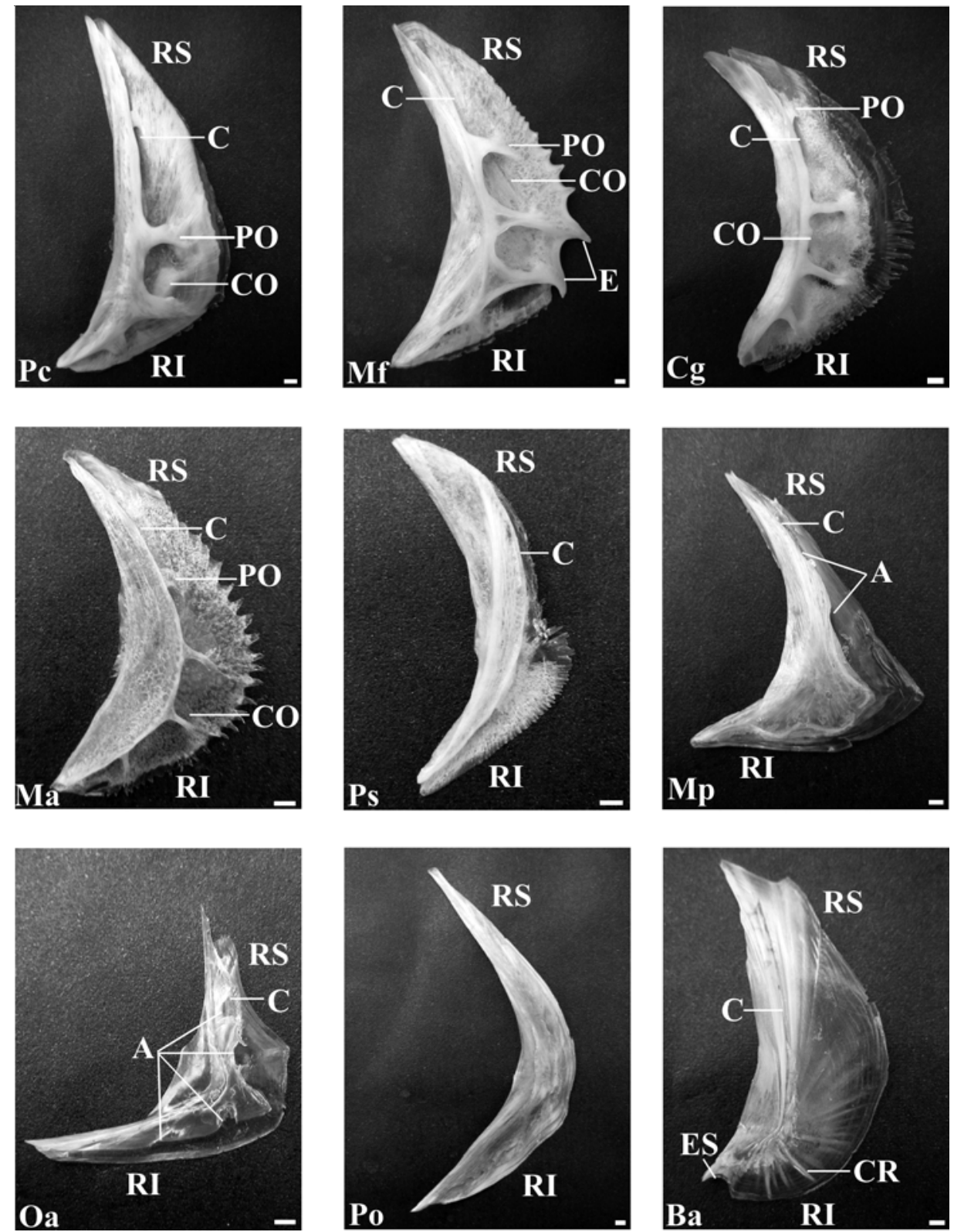

Figura 11

Fotografías del preopérculo en cara lateral externa para las 9 especies de peces analizadas. Abreviaturas: A: aberturas; C: canal línea lateral; CO: concavidad; CR: canales radiales; E: espinas; ES: escotadura; PO: puente óseo; RI: rama inferior; RS: rama superior. Escala: $3 \mathbf{~ m m}$

Photographies of the preopercle in lateral outer side for the 9 fish species analyzed. Abbreviations: A: openings; C: laterosensory canal; CO: concavity; CR: radial channels; E: spines; ES: recess; PO: osseous bridge; RI: lower branch; RS: upper branch. Scale bar: $3 \mathrm{~mm}$

\section{Postemporal}

El postemporal presenta la típica forma de horquilla, con dos ramas anteriores y una superficie posterior expandida (Fig.12). La rama superior, en todas las especies analizadas, es más larga que la inferior y es aguzada, con su extremo anterior en punta, excepto en $P$. orbignyanus, cuya rama superior es ancha en toda su extensión. La rama inferior es muy corta en comparación con la superior en $P$. orbignyanus; es casi tan larga como la superior en B. aurea; y es corta en las demás especies analizadas. En su base se sitúa una saliente espinosa muy desarrollada en $M$. americanus, poco desarrollada en $M$. furnieri y 

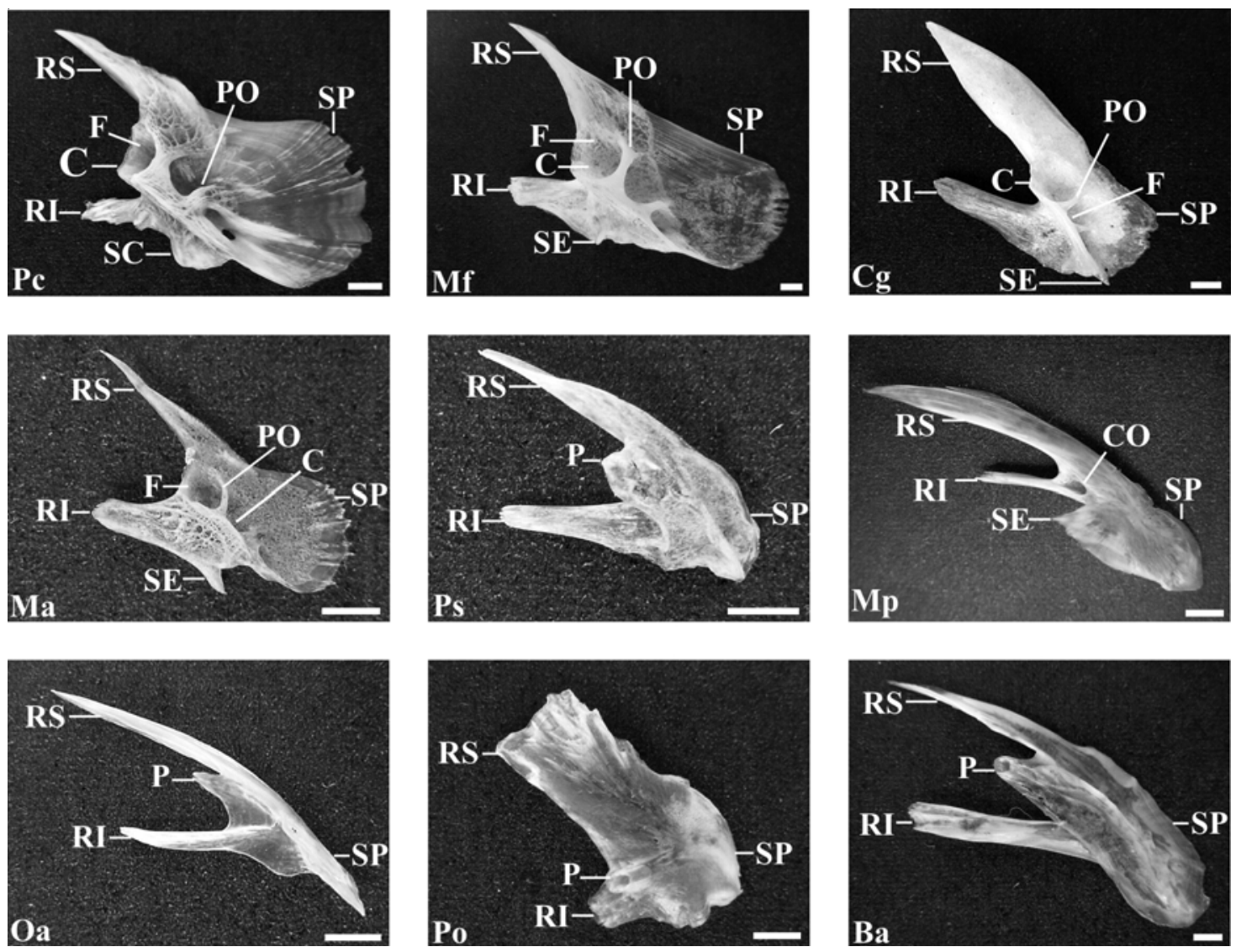

Figura 12

Fotografías del postemporal en cara lateral externa para las 9 especies de peces analizadas. Abreviaturas: C: canal línea lateral; CO: concavidad; F: fenestra; P: proceso; PO: puente óseo; RI: rama inferior; RS: rama superior; SC: saliente convexa; SE: saliente espinosa; SP: superficie posterior. Escala: $\mathbf{3} \mathbf{~ m m}$

Photographies of the posttemporal in lateral outer side for the 9 fish species analyzed. Abbreviations: C: laterosensory canal; CO: concavity; F: fenestra; P: process; PO: osseous bridge; RI: lower branch; RS: upper branch; SC: convex salient; SE: thorny salient; SP: posterior surface. Scale bar: $3 \mathrm{~mm}$

una saliente convexa de base ancha en $P$. cromis.

La longitud de la superficie posterior (medida desde el punto de inflexión entre las ramas hasta su extremo posterior) es mayor a la longitud de la rama superior (medida desde su extremo al punto de inflexión entre las ramas) en $M$. americanus (rango 64-98\%, media 82), $M$. furnieri (rango 69-107\%, media 85), P. orbignyanus (rango 75-136\%, media 90) y P. cromis (rango 82-105\%, media 91). Es menor en M. platanus (rango 111-169\%, media 128), O. argentinensis (rango 131-155\%, media 145), $P$. saltatrix (rango 128-165\%, media 151), C. guatucupa (rango 139-176\%, media 156) y B. aurea (rango 138-205\%, media 172).

El borde de la superficie posterior es redondeado e irregular en todas las especies estudiadas, excepto en $O$. argentinensis, en el que termina en punta. En M. platanus se extiende en un proceso espinoso anterior. En $C$. guatucupa presenta una pequeña saliente espinosa posterior.

En las especies de la Familia Sciaenidae, a lo largo del postemporal, se extiende un canal externo correspondiente al conducto de la línea lateral, interrumpido por dos puentes óseos que delimitan tres fenestras, la primera de las cuales se sitúa entre las ramas del postemporal. En el resto de las especies el canal es interno. Entre las ramas del postemporal en $M$. platanus se encuentra una concavidad somera, mientras que en las demás especies se sitúa un proceso. En $P$. saltatrix el proceso es corto y triangular. En O. argentinensis es largo y espinoso. En B. aurea es largo y presenta un gran orificio en su extremo. En P. orbignyanus es muy corto y también exhibe un orificio en su extremo. 


\section{Claves diagnósticas}

\section{Clave paraesfenoides (Fig. 1)}

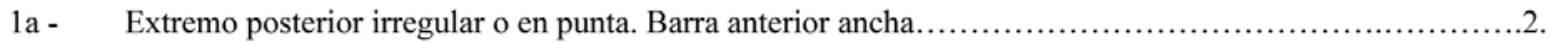

$1 \mathrm{~b}$ - $\quad$ Extremo posterior escindido en dos procesos espinosos. Barra anterior muy delgada.......................

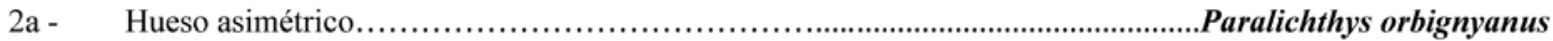

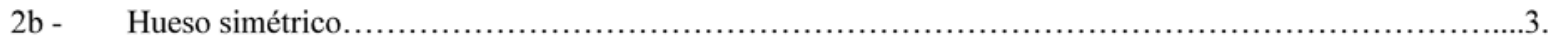

3a - Apófisis alares situadas hacia la mitad del hueso. Barra anterior, terminada en dos pequeñas puntas.

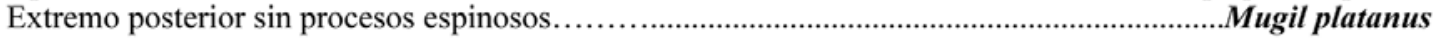

3b - Apófisis alares situadas hacia el extremo posterior del hueso. Barra anterior, terminada en una punta.

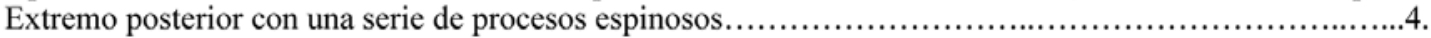

4a - Procesos espinosos cortos.

Cynoscion guatucupa

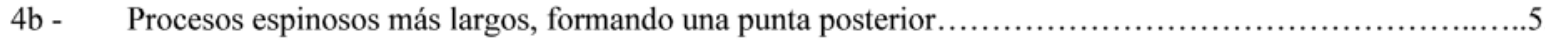

5a - Bordes laterales de la barra anterior, rectos hacia el extremo anterior, luego convexos y posteriormente, cóncavos. Apófisis alares de base ancha y bordes cóncavos.

Menticirrhus americanus

$5 \mathrm{~b}$ - Bordes laterales de la barra anterior ligeramente cóncavos o rectos. Apófisis alares de base angosta y redondeadas. . .

6a- Superficie posterior de bordes convexos, con una cresta perpendicular que la separa de la barra anterior......

Pogonias cromis

6b - Superficie posterior de bordes cóncavos, sin cresta.

Micropogonias furnieri

7a - Superficie posterior de bordes laterales rectos y terminada en dos proyecciones muy

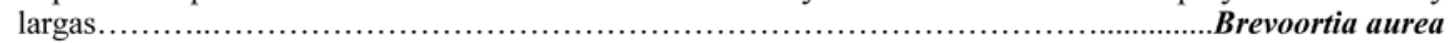

7b - Superficie posterior de bordes laterales convexos y terminada en dos proyecciones espinosas

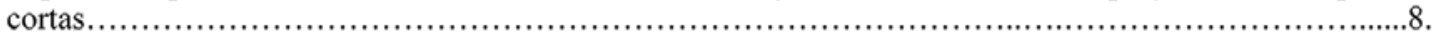

8a- Apófisis alares triangulares. Superficie posterior corta. La barra anterior termina en un borde irregular.

Odontesthes argentinensis

8b - Apófisis alares redondeadas. Superficie posterior alargada. La barra anterior termina en un borde redondeado.

Pomatomus saltatrix

Clave lacrimal (Fig. 2)

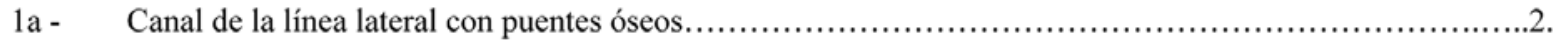

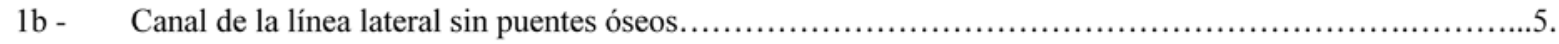

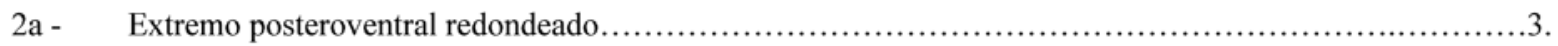

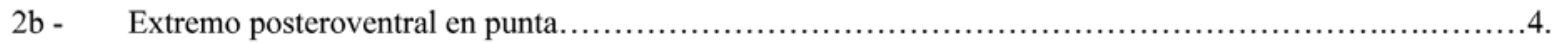

$3 a$ - Puentes óseos gruesos. Abertura superior circular. Margen inferior liso................................Pogonias cromis

3b - Puentes óseos delgados. Abertura superior aproximadamente triangular. Margen inferior aserrado........................................................................................enticirrhus americanus

4a - Hueso expandido dorsoventralmente. Extremo posterodorsal de base ancha. Con proyección dorsal

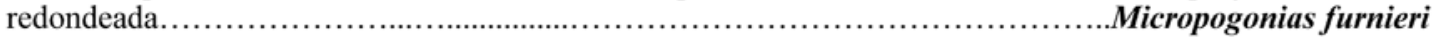

4b- Hueso deprimido dorsoventralmente. Extremo posterodorsal de base angosta. Sin proyección

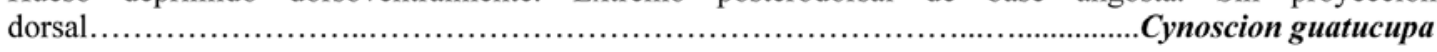

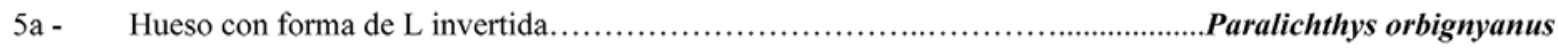

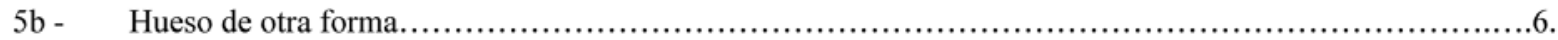

6a- Canal principal situado en el borde superior del hueso, del que surgen tres canales

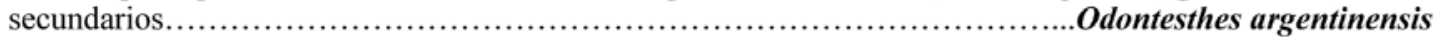

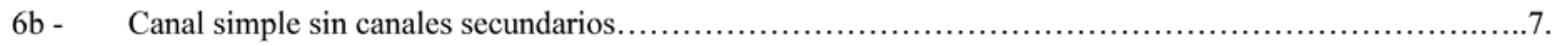

7a - Extremo anterior con dos procesos espinosos y posterior redondeado..............................Mugil platanus

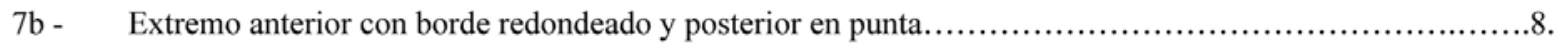

8a - Borde inferior liso. Saliente dorsal redondeada. Canal de la línea lateral situado en el margen superior del hueso.

Brevoortia aurea 
8b - Borde inferior aserrado. Sin saliente dorsal. Canal de la línea lateral situado en el margen inferior del hueso.

Clave premaxilar (Figs. 3 y 4 )

1a - Premaxilar muy reducido, sin procesos y sin dientes

Brevoortia aurea

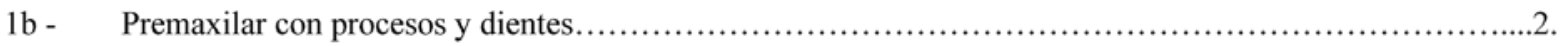

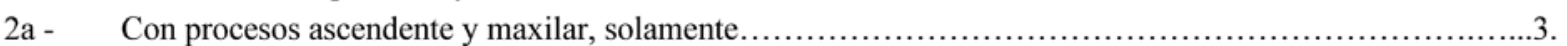

$2 \mathrm{~b}$ - Con procesos ascendente, articular y maxilar...............................................................

3a - Proceso ascendente situado anteriormente, vertical, de forma rectangular y con un orificio central. El proceso maxilar se proyecta dorsalmente en el extremo posterior del hueso y presenta forma triangular..... Mugil platanus

3b - Proceso ascendente situado hacia la mitad del hueso, alargado y curvado anteriormente. El proceso maxilar de borde redondeado, se encuentra en el extremo posterior del hueso y se expande dorsal y ventralmente. Odontesthes argentinensis

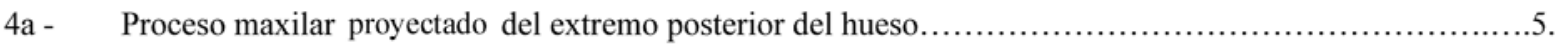

$4 \mathrm{~b}$ - $\quad$ Proceso maxilar proyectado anteriormente al extremo posterior del hueso.................................

5a - Dientes cónicos y grandes, dispuestos en una fila en el borde externo, posteriormente seguida por varias filas de dientes viliformes muy pequeños. Menticirrhus americanus

$5 \mathrm{~b}$ - $\quad$ Dientes viliformes dispuestos en varias filas de manera desordenada....................................

6a - Longitud del proceso ascendente menor a la longitud total del hueso. Internamente, el proceso ascendente y el articular se encuentran separados por una escotadura en forma de $\mathrm{V}$ que se inicia más arriba de la base del proceso articular.

Micropogonias furnieri

6b - Longitud del proceso ascendente mayor a la longitud total del hueso. Internamente, el proceso ascendente y el articular se encuentran separados por una escotadura fina y profunda, que se inicia a la altura de la base del proceso articular.

Pogonias cromis

7a - Proceso maxilar proyectado dorsalmente hacia la mitad del hueso. Proceso articular muy inclinado posteriormente y separado del proceso ascendente. Dientes caniniformes ...........Paralichthys orbignyanus

7b - Proceso maxilar proyectado dorsalmente hacia el tercio posterior del hueso. Proceso articular se sitúa

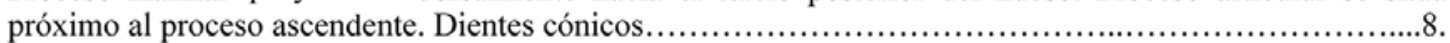

8a- Dientes muy numerosos $(>100)$, dispuestos anteriormente en tres filas y posteriormente en dos filas.

Cynoscion guatucupa

8b - Dientes numerosos $(<30)$, dispuestos en una fila. En la cara lateroventral interna presenta una segunda fila de dientes muy pequeños que se extiende hasta aproximadamente la mitad de la longitud del hueso

Pomatomus saltatrix

Clave dentario (Fig. 5)

1a - Surco profundo en la superficie lateroventral, que recorre toda la longitud del hueso y presenta puentes

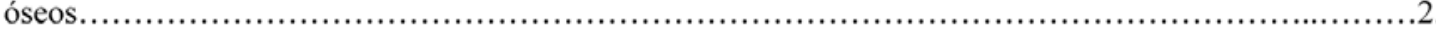

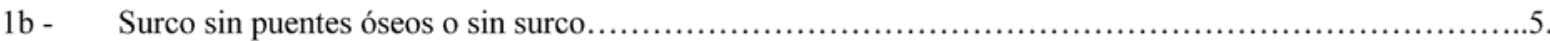

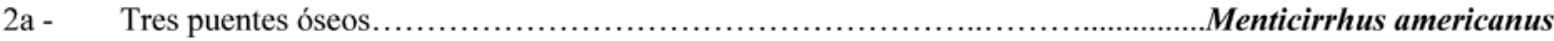

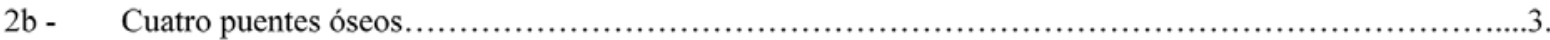

3a - Presencia de un poro muy pequeño hacia la mitad de la superficie laterodorsal. Dientes numerosos (55-85), cónicos, curvados hacia el interior de la boca. Se disponen, anteriormente en tres filas y posteriormente en dos filas....

Cynoscion guatucupa

3b - Presencia de un gran orificio situado en la porción anterior de la superficie laterodorsal. Dientes muy numerosos (150-180), viliformes, ligeramente curvados hacia el interior de la boca. Se disponen, anteriormente en varias filas desordenadas y posteriormente se reducen en número hasta constituir una única hilera.

4a - Rama inferior redondeada posteriormente. Los dientes se reducen en número y constituyen una única fila hacia aproximadamente la mitad del hueso

Pogonias cromis

$4 \mathrm{~b}$ - $\quad$ Rama inferior espinosa posteriormente. Los dientes se reducen en número y constituyen una única fila hacia aproximadamente la base de la rama superior... Micropogonias furnieri 
5a - Rama superior muy ancha y de borde redondeado. Rama inferior espinosa....

$5 \mathrm{~b}$ - $\quad$ Rama superior delgada y de borde aproximadamente rectangular. Rama inferior terminada en dos puntas o en borde irregular.

$7 \mathrm{~b}$ - Rama superior alta. La rama inferior termina en borde irregular.

Rama superior curvada hacia el interior de la boca. En la cara laterodorsal y hacia la rama inferior presenta dos aberturas ovaladas. Numerosos dientes muy finos y largos, dispuestos en una fila formando una banda.

Mugil platanus

8b - Rama superior no curvada. En la cara lateroventral se sitúa un surco somero. Dientes poco numerosos, cónicos, dispuestos en una fila muy separados entre sí.

Pomatomus saltatrix

Clave anguloarticular (Fig. 6)

1a - Canal de la línea lateral con un puente óseo.

$1 \mathrm{~b}-$

Canal de la línea lateral interno sin puentes óseos.

$2 \mathrm{~b}$ - Proceso anterior espinoso y corto. Retroarticular con forma de espina situado en la base del puente óseo.

Pogonias cromis

3a - Puente óseo muy ancho 4.

3b - Puente óseo delgado.

4a - Canal de la línea lateral muy ancho. Proceso coronoides de borde dorsal ondulado...Micropogonias furnieri

$4 \mathrm{~b}-$ Canal de la línea lateral angosto. Proceso coronoides de borde dorsal convexo. .Menticirrhus americanus

$5 \mathrm{a}-$ Proceso coronoides delgado. Proceso vertebral plano. Brevoortia aurea

$7 \mathrm{~b}$ - Proceso posterior curvado hacia arriba y redondeado. Proceso anterior prominente con gran abertura dorsal. Odontesthes argentinensis

8a - Proceso posterior recto y en punta. Superficie ventral cuadrangular. Proceso anterior muy pequeño y espinoso Mugil platanus

$8 \mathrm{~b}$ - Proceso posterior curvado y redondeado. Superficie ventral rectangular. Proceso anterior grande y redondeado. . .

Pomatomus saltatrix

Clave palatino (Fig. 7)

1a - Palatino con extremo posterior ancho, no espinoso.................................................................Brevoortia aurea

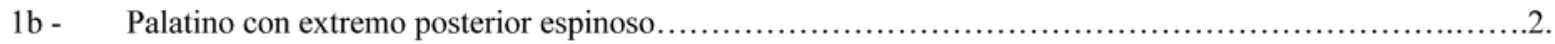

2a - Superficie ventral con dientes.

Pomatomus saltatrix

$2 \mathrm{~b}-$

Superficie ventral sin dientes.

Presencia de una fuerte cresta que forma con la superficie ventral una gran concavidad.......................4.

$3 b-$

Ausencia de cresta y concavidad. 
7a - Saliente ubicada en el borde superior del proceso anterior, triangular y muy grande. En el borde superior, hacia la mitad del hueso, se sitúa un proceso en forma de varilla. Odontesthes argentinensis

$7 \mathrm{~b}$ - Saliente ubicada en el borde superior del proceso anterior, redondeada y pequeña. Proceso

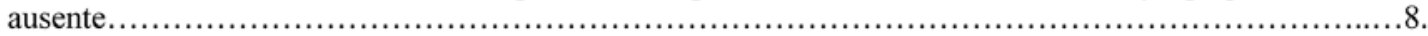

8a - Superficie ventral de borde triangular.

Mugil platanus

8 b - Superficie ventral de borde recto Paralichthys orbignyanus

\section{Clave hiomandibular (Fig. 8)}

1a - Apófisis auricular con una profunda hendidura en forma de V. Gran orificio ovalado en la base superior del eje vertical. Expansión anterior curvada hacia arriba, terminada en un apéndice espinoso..Brevoortia aurea

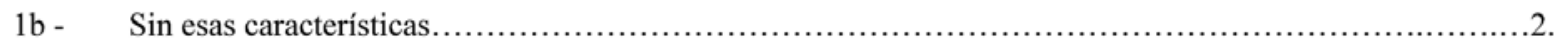

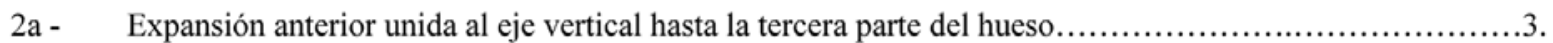

$2 b$ - Expansión anterior unida al eje vertical hasta la primera parte o hasta la mitad del hueso......................

3a - La cresta comienza y finaliza en un proceso espinoso. Expansión anterior de forma

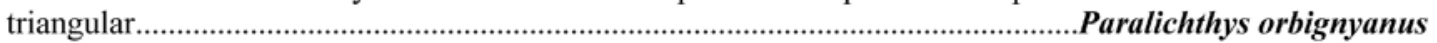

3b - $\quad$ La cresta no presenta procesos espinosos. Expansión anterior de otra forma.................................................4.

4a - Expansión de forma aproximadamente rectangular y borde irregular, separada del eje vertical por una escotadura en forma de U. Odontesthes argentinensis

4b - Expansión anterior de forma redondeada y borde liso, sin escotadura. Pomatomus saltatrix

5a - Expansión anterior de borde festoneado Mugil platanus

5 b - Expansión anterior de borde irregular. 6.

6a - Proyección espinosa dorsal relacionada a la apófisis opercular a través de una lámina recta. La cresta longitudinal se bifurca inferiormente en dos ramas que delimitan un canal.................................

6b - Proyección espinosa dorsal relacionada a la apófisis opercular a través de una lámina convexa. La cresta longitudinal se bifurca inferiormente en dos ramas que delimitan una concavidad amplia..................8.

7a - Expansión anterior de forma triangular. Proyección espinosa delgada. Apófisis opercular

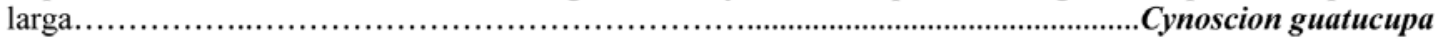

7b - Expansión anterior de forma redondeada. Proyección espinosa gruesa. Apófisis opercular

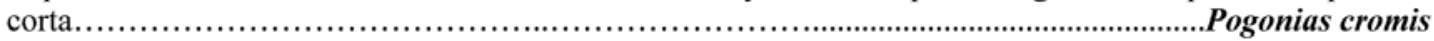

8a - Rama anterior de la cresta curvada y paralela al eje vertical. Concavidad divida por un tabique óseo. Expansión anterior larga.

Micropogonias furnieri

8b - Rama anterior de la cresta aproximadamente recta y perpendicular al eje vertical. Concavidad sin tabiques óseos. Expansión anterior corta. Menticirrhus americanus

\section{Clave ceratohial (Fig. 9)}

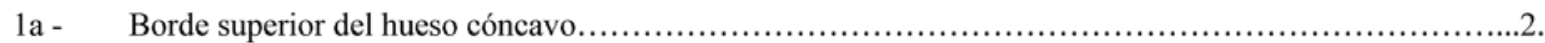

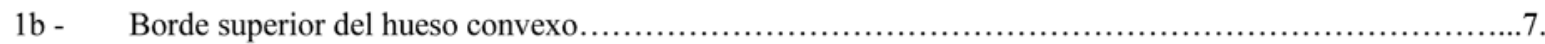

2a - Borde inferior del hueso cóncavo. Extremo posterior expandido inferiormente.............................

$2 \mathrm{~b}$ - $\quad$ Borde inferior ondulado. Extremo posterior expandido tanto inferior como superiormente....................

3a - Extremo anterior ligeramente expandido y redondeado. Porción posteroinferior aproximadamente rectangular, sin concavidades.

Odontesthes argentinensis

3b - Extremo anterior muy expandido y cóncavo. Porción posteroinferior aproximadamente cuadrangular, con dos concavidades someras.

Mugil platanus

4a - Extremo posteroinferior redondeado

Cynoscion guatucupa

$4 b-$

Extremo posteroinferior en punta.

. .5 .

5a - Porción posteroinferior con dos concavidades bien delimitadas. Los procesos espinosos se extienden del borde posterior en la cara interna.

Pogonias cromis

$5 \mathrm{~b}$ - Porción posteroinferior con concavidades poco definidas. Los procesos espinosos se extienden del borde

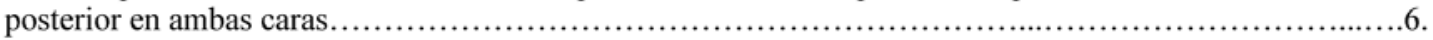

6a - Extremo anterior con proyección inferior larga.

Micropogonias furnieri 
6b - Extremo anterior con proyecciones cortas.

Menticirrhus americanus

7a - Porción posteroinferior triangular. Borde inferior con dos concavidades y un proceso espinoso

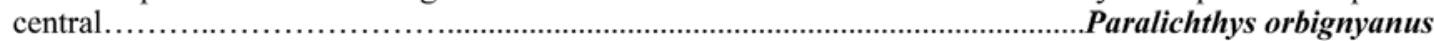

$7 \mathrm{~b}$ - Porción posteroinferior cuadrangular, curvado anteriormente en un pequeño proceso espinoso. Borde

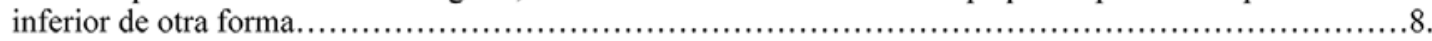

8a - Borde inferior ondulado. Extremo anterior en forma de varilla delgada y corta. En el centro del hueso se sitúa una fosa alargada.

Pomatomus saltatrix

$8 \mathrm{~b}$ - Borde inferior irregular con cuatro orificios. Extremo anterior ancho y redondeado. En la porción posterior del hueso se sitúa un orificio ovalado...

Brevoortia aurea

Clave urohial (Fig. 10)

1a - Hueso curvado con vértice dirigido hacia atrás y dos brazos anteriores..................Paralichthys orbignyanus

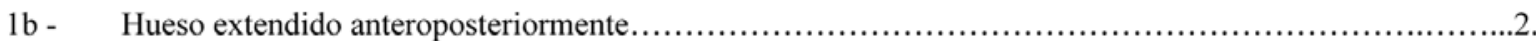

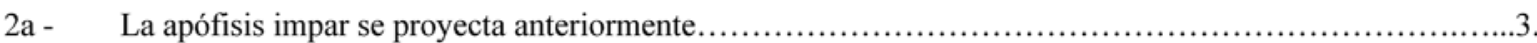

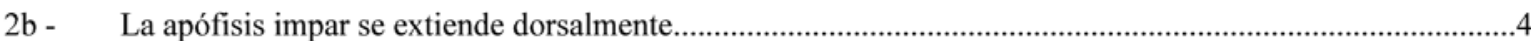

3a - Hueso muy largo, comprimido dorsoventralmente y curvado hacia arriba............................Brevoortia aurea

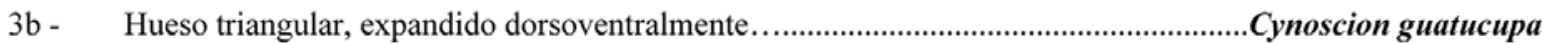

4a - Extremo posteroinferior del hueso bifurcado en dos salientes espinosas. Lámina ventral

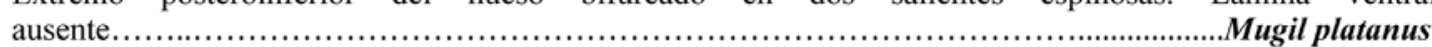

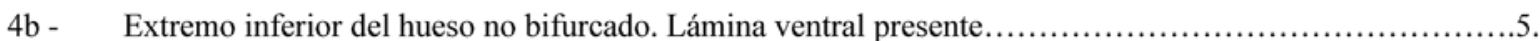

5a - Lámina dorsal muy expandida. La apófisis impar se continúa con la lámina

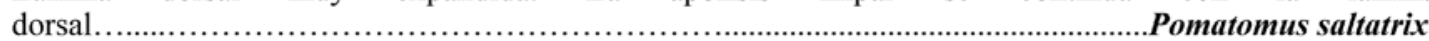

5b - Lámina dorsal más angosta. La apófisis impar se separa de la lámina dorsal.

..6.

6a - Extremo posterosuperior del hueso bifurcado en dos apófisis espinosas. Borde inferior de la lámina ventral expandido en dos prolongaciones laterales, en forma de pequeñas alas.

Odontesthes argentinensis

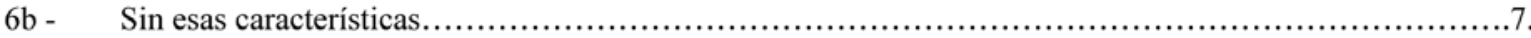

7a - Lámina ventral de borde posterior convexo..................................................................enticirrhus americanus

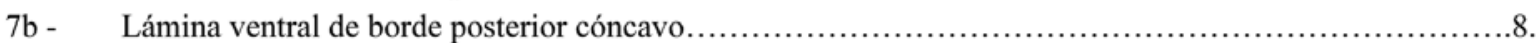

8a - Apófisis impar pequeña. Extremo posterosuperior del hueso terminado en una punta muy

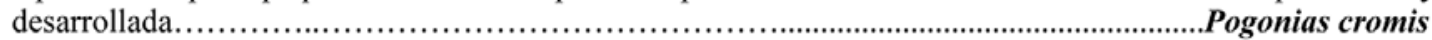

8b - Apófisis impar prominente. Extremo posterosuperior del hueso terminado en una pequeña

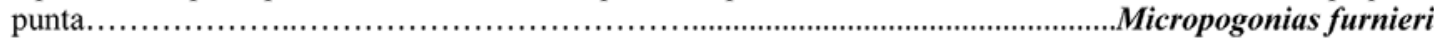

Clave preopérculo (Fig. 11)

1a - Con canal o surco longitudinal con puentes óseos. $\quad$.

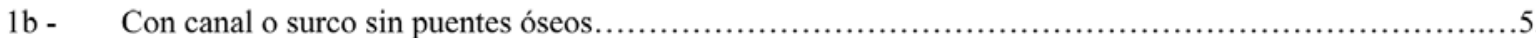

2a - Borde posterior con varias proyecciones espinosas pequeñas y en el punto de curvatura, dos espinas muy desarrolladas

Micropogonias furnieri

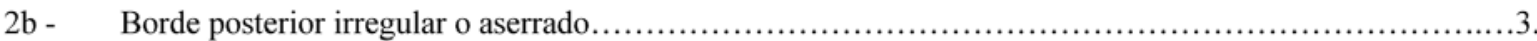

3a- Borde posterior irregular. Puentes óseos anchos, que delimitan concavidades aproximadamente ovaladas. ...

Pogonias cromis

$3 \mathrm{~b}$ - Borde posterior aserrado. Puentes óseos delgados, que delimitan concavidades rectangulares...............4.

4a - Algunos de los puentes óseos terminan cerca del borde posterior y se inician debajo del borde anterior. Borde anterior ancho.

Menticirrhus americanus

4b - Los puentes óseos se continúan del borde anterior y terminan lejos del borde posterior. Borde anterior delgado.

Cynoscion guatucupa

5a - Canal cerrado, sólo abre mediante un poro en cada extremo del hueso................Paralichthys orbignyanus

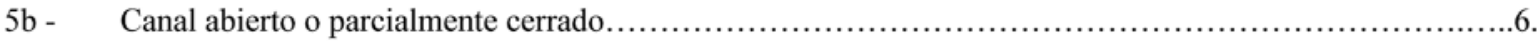

6a - Canal abierto, delgado y en su región ventral se ramifica en pequeños canales radiales. El extremo inferior en punta es seguido por una pequeña escotadura.

Brevoortia aurea 
6b - Canal parcialmente cerrado. El extremo inferior no presenta esa característica. 7.

$7 \mathrm{a}$ - Canal parcialmente abierto a lo largo de ambas ramas. Las ramas presentan similar longitud. Odontesthes argentinensis

$7 \mathrm{~b}$ - $\quad$ Canal cerrado a lo largo de una de las ramas. La rama superior es más larga que la inferior..................

8a - Hueso largo y angosto en el punto de curvatura. Borde posterior aserrado y

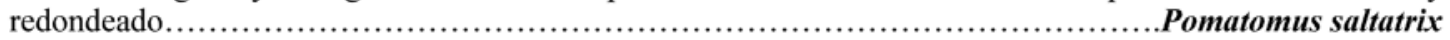

$8 \mathrm{~b}$ - Hueso corto y ancho en el punto de curvatura. Borde posterior liso y en punta........ Mugil platanus

Clave postemporal (Fig. 12)

1a - Rama superior ancha en toda su extensión. Rama inferior muy corta, en comparación a la superior...... Paralichthys orbignyanus

1b - Rama superior aguzada y terminada en punta. Rama inferior más larga.....................................

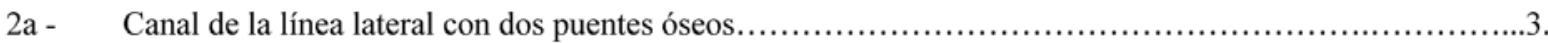

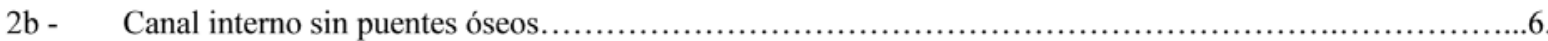

3a - Longitud de la superficie posterior menor a la longitud de la rama superior. Borde de la superficie posterior con una pequeña saliente espinosa.

Cynoscion guatucupa

3b - Longitud de la superficie posterior mayor a la longitud de la rama superior. El borde de la superficie posterior no presenta una saliente espinosa. ..4.

4a - En la base de la rama inferior se sitúa una saliente convexa.

Pogonias cromis

$4 \mathrm{~b}$ - $\quad$ En la base de la rama inferior se sitúa una saliente espinosa.

\section{.5 .}

5a - Saliente espinosa grande. Borde superior de la superficie posterior anteriormente cóncavo y posteriormente convexo. Menticirrhus americanus

$5 \mathrm{~b}$ - $\quad$ Saliente espinosa pequeña. Borde superior de la superficie posterior recto Micropogonias furnieri

6a - Entre ambas ramas se sitúa una concavidad somera. Del borde inferior de la superficie posterior se extiende un proceso espinoso, dirigido anteriormente..... Mugil platanus

$6 \mathrm{~b}$ - $\quad$ Entre ambas ramas se sitúa un proceso. Del borde inferior no se extiende un proceso........................

$7 \mathrm{a}$ - Entre ambas ramas se encuentra un proceso largo, con un gran orificio en su extremo........Brevoortia aurea

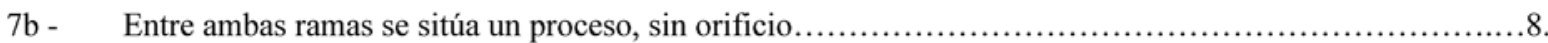

$8 \mathrm{a}$ - $\quad$ Proceso corto y triangular. Superficie posterior de borde redondeado.........................Pomatomus saltatrix

$8 \mathrm{~b}$ - $\quad$ Proceso alargado y espinoso. Superficie posterior terminada en punta................... Odontesthes argentinensis

\section{Agradecimientos}

Los autores agradecen al grupo de investigación Ictiología, del Depto. de Ciencias Marinas, por el apoyo brindado durante la colecta del material. Este trabajo fue financiado a través de fondos de la Comisión de Investigaciones Científicas de la Provincia de Buenos Aires (Programa de subsidios para proyectos de investigación, desarrollo y transferencia, 0823/07). Agradecemos a evaluadores anónimos, cuyos comentarios y sugerencias contribuyeron a mejorar sustancialmente el manuscrito.

\section{Literatura citada}

Abruzzini AC. 2002. Los peces juveniles de la laguna Mar Chiquita. Tesis de grado, Facultad de Ciencias Exactas y Naturales, Universidad Nacional de Mar del Plata, Mar del Plata, Argentina, 42 pp.
Alexander RM. 1967. Mechanisms of the jaws of some atheriniform fish. Journal of Zoology 151: 233-255.

Azpelicueta MM, DE Figueroa, JM Díaz de Astarloa \& MB Cousseau. 1998. Freshwater fishes in a world reserve of biosphere: Mar Chiquita coastal lagoon (Buenos Aires, Argentina). Biogeographica 74: 85-90.

Bemvenuti MA. 1995. Odontesthes mirinensis sp. n., um novo peixe-rei (Pisces, Atherinidae, Atherinopsinae) para o extremo sul do Brasil. Revista Brasileira de Zoología 12: 881-903.

Bemvenuti MA. 1997. Relações morfológicas e osteológicas entre Odontesthes perugiae e O. mirinensis (Teleostei: Atherinidae, Atherinopsinae). Atlántica 19: 113-131.

Bó MS, JP Isacch, AI Malizia \& MM Martínez. 2001. Lista de mamíferos de la reserva Mar Chiquita. En: Iribarne O (ed). Reserva de biosfera Mar Chiquita: Características físicas, biológicas y ecológicas, pp. 303-309. Editorial Martín, Mar del Plata. 
Borodulina OD. 1984. Identification of remains of mesopelagic fishes from stomachs of predators. Report III. The structure of jaws of common stomiatoid fishes of the families Gonostomatidae, Sternoptychidae and Photichthyidae. Journal of Ichthyology 24: 103-111.

Campello FD \& MA Bemvenuti. 2002. Diferenciação morfométrica e osteológica entre Ramnogaster arcuata (Jenyns) e Platanichthys platana (Regan) (Teleostei, Clupeidae). Revista Brasileira de Zoología 19: 757-766.

Cerdá Gilbert MA 1999. Los pejerreyes de la laguna Mar Chiquita: sistemática y estructura poblacional. Tesis de grado, Facultad de Ciencias Exactas y Naturales. Universidad Nacional de Mar del Plata, Mar del Plata, Argentina, 33 pp.

Cervigón F. 1980. Ictiología marina. 1: 1-358. Editorial Arte, Caracas.

Chapleau F. 1993. Pleuronectiform relationships: a cladistic reassessment. Bulletin of Marine Science 52: 516-540.

Collette BB \& GB Gillis. 1992. Morphology, systematics and biology of the double-lined mackerels (Grammatorcynus, Scombridae). Fishery Bulletin 90: 13-53.

Cousseau MB, JM Díaz de Astarloa \& DE Figueroa. 2001. La ictiofauna de la laguna Mar Chiquita. En: Iribarne O (ed). Reserva de biosfera Mar Chiquita: Características físicas, biológicas y ecológicas, pp. 187-204. Editorial Martín, Mar del Plata.

Cousseau MB \& RG Perrotta. 2004. Peces marinos de Argentina. Biología, distribución, pesca, 167 pp. Instituto Nacional de Investigación y Desarrollo Pesquero, Mar del Plata.

Cousseau MB, AE Gosztonyi, I Elías \& ME Ré. 2004. Estado actual del conocimiento de los peces de la plataforma argentina y adyacencias. En: Sánchez RP \& SI Bezzi (eds). El Mar Argentino y sus recursos pesqueros. Tomo 4. Los peces marinos de interés pesquero. Caracterización biológica y evaluación del estado de explotación, pp. 1738. Instituto Nacional de Investigación y Desarrollo Pesquero, Mar del Plata.

De Francesco CG \& FI Isla. 2001. Gasterópodos bioindicadores de salinidad en Mar Chiquita. En: Iribarne O. (ed). Reserva de biosfera Mar Chiquita: Características físicas, biológicas y ecológicas, pp. 115-119. Editorial Martín. Mar del Plata.

Díaz de Astarloa JM. 1991. Estudios osteológicos del sincráneo y complejo caudal en dos formas nominales de Paralichthys: Paralichthys patagonicus y Paralichthys bicyclophorus. Frente Marítimo 9: 15-27.

Díaz de Astarloa JM. 1994. Las especies del género Paralichthys (Pisces, Paralichthyidae) del Mar Argentino. Morfología y Sistemática. Tesis Doctoral, Universidad Nacional de Mar del Plata, Mar del Plata, 194 pp.

Díaz de Astarloa JM. 2005. Osteología craneal comparada de tres especies de lenguado del género Paralichthys (Pleuronectiformes, Paralichthyidae) del Atlántico suroccidental. Revista Chilena de Historia Natural 78: 343391.

Díaz de Astarloa JM, A Aubone \& MB Cousseau. 1999. Asociaciones ícticas de la plataforma costera de Uruguay y norte de Argentina y su relación con los parámetros ambientales. Physis 57: 29-45.

Díaz de Astarloa JM, DE Figueroa, MB Cousseau \& M Barragán. 2000. Occurrence of Trachinotus carolinus (Carangidae) in Laguna Costera Mar Chiquita, with comments on other occasionally recorded fishes in Argentinean waters. Bulletin of Marine Science 66: 399404.

Dyer B. 2006. Systematic revision of the South American silversides (Teleostei, Atheriniformes). Biocell 30: 69-88.

Favero M, S Bachmann, S Copello, R Mariano-Jelicich, MP Silva, M Ghys, C Khatchikian \& L Mauco. 2001. Aves marinas del sudeste bonaerense. En: Iribarne O. (ed). Reserva de biosfera Mar Chiquita: Características físicas, biológicas y ecológicas, pp. 251-267. Editorial Martín, Mar del Plata.

Ferrero L. 2001. Avifauna de Mar Chiquita. Síntesis de la tesis Doctoral de M.M. Martínez. En: Iribarne O (ed). Reserva de biosfera Mar Chiquita: Características físicas, biológicas y ecológicas, pp. 227-250. Editorial Martín, Mar del Plata.

Figueroa DE, JM Díaz de Astarloa \& MB Cousseau. 2000. Southernmost occurrence of the aguavina on the western Atlantic coast of Argentina. Journal of Fish Biology 56: $1280-1282$.

González Castro M, JM Díaz de Astarloa \& MB Cousseau. 2006. First record of a tropical affinity mullet, Mugil curema (Actinopterygii: Mugilidae), in a temperate Southwestern Atlantic coastal lagoon. Cybium 30: 90-91.

González Castro M. 2007. Los peces representantes de la Familia Mugilidae en Argentina. Tesis Doctoral, Universidad Nacional de Mar del Plata, Mar del Plata, 182 pp.

Gosztonyi AE \& L Kuba. 1996. Atlas de huesos craneales y de la cintura escapular de peces costeros patagónicos. Fundación Patagonia Natural, Informe Técnico 4: 1-29.

Granadeiro JP \& Silva MA. 2000. The use of otoliths and vertebrae in the identification and size-estimation of fish in predator-prey studies. Cybium 24: 383-393.

Greenwood PH, DE Rosen, SH Weitzman \& GS Myers. 1966. Phyletic studies of teleostean fishes, with a provisional classification of living forms. Bulletin of the American Museum of Natural History 131: 341-455.

Greenwood PH, RS Miles \& C Patterson. 1973. Interrelationships of Fishes. Zoological Journal of the Linnean Society 53: 536.

Hansel HC, SD Dyke, PT Lofy \& GA Gray. 1988. Use of diagnostic bones to identify and estimate original lengths of ingested preys fishes. Transactions of the American Fisheries Society 117: 55-62. 
Heras S, M González Castro \& MI Roldán. 2006. Mugil curema in Argentinean waters: combined morphological and molecular approach. Aquaculture 261: 473-478.

Hoshino K. 2001. Monophyly of the Citharidae (Pleuronectoidei: Pleuronectiformes: Teleostei) with considerations of pleuronectoid phylogeny. Ichthyological Research 48: 391-404.

Hozbor NM \& SB García de la Rosa. 2000. Alimentación de juveniles de corvina rubia (Micropogonias furnieri) en la laguna costera Mar Chiquita (Buenos Aires, Argentina). Frente Marítimo 18: 59-70.

Iribarne $O$ (ed). 2001. Reserva de Biosfera Mar Chiquita: Características físicas, biológicas y ecológicas. 320 pp. Editorial Martín, Mar del Plata.

Jardim LF. 1988. Osteologia do sincrãnio das espécies de Menticirrhus Gill, 1861 da Costa Sul do Brasil (Perciformes, Sciaenidae). Atlántica 10: 41-74.

Kong I \& P Iratchet. 1995. Osteología comparada del cráneo de ocho especies de peces Sciaenidae de Chile. Estudios Oceanológicos 14: 5-16.

Lloris D \& J Matallanas. 2003. Description of a new species of hake: Merluccius patagonicus sp. nov. (Gadiformes, Merlucciidae) from the waters of Argentina. Scientia Marina 67: 323-326.

Lucifora LO \& RC Menni. 1998. First record of a porbeagle shark, Lamna nasus, in brackish waters of Mar Chiquita lagoon, Argentina. Cybium 22: 87-88.

Malizia AI, D Antinuchi \& AI Vasallo. 2001. Aspectos ecológicos de la comunidad de roedores de la reserva de Mar Chiquita con énfasis en el roedor subterráneo Ctenomys talarum. En: Iribarne O (ed). Reserva de biosfera Mar Chiquita: Características físicas, biológicas y ecológicas, pp. 287-301. Editorial Martín, Mar del Plata.

Matallanas J \& Lloris D. 2006. Description of Merluccius tasmanicus sp. Nov. and redescription of Merluccius australis (Pisces: Merluciidae). Journal of the Marine Biological Association of the United Kingdom 86: 193199.

Monod T. 1968. Le complexe urophore des poissons téleóstéens. Mémoires de I'Institut Fondamental d'Afrique Noire 81: 1-705.

Newsome GE. 1977. Use of opercular bones to identify and estimate lengths of prey consumed by piscivores. Canadian Journal of Zoology 55: 733-736.

Nybelin O. 1973. Comments on the caudal skeleton of Actinopterygians, En: Greenwood PH, RS Miles \& C
Patterson (eds). Interrelationships of fishes. Zoological Journal of the Linnean Society 53: 369-372.

Piacentino GL. 1999. Osteología craneana de Odontesthes nigricans (Richardson 1845) y Odontesthes smitti (Lahille 1929) de la Ría de Puerto Deseado (Santa Cruz, Argentina) (Teleostei, Atherinopsidae). Boletim do Laboratório de Hidrobiología, São Luis 12: 23-47.

Pietsch TW \& CP Zabetian. 1990. Osteology and interrelationships of the sand lances (Teleostei: Ammodytidae). Copeia 1990: 78-100.

Rivera Prisco A, SB García de la Rosa \& JM Díaz de Astarloa. 2001. Feeding ecology of flatfish juveniles (Pleuronectiformes) in Mar Chiquita coastal lagoon (Buenos Aires, Argentina). Estuaries 24: 917-925.

Rojo AL. 1988. Diccionario enciclopédico de anatomía de peces. Monografías del Instituto Español de Oceanografía $\mathrm{N}^{\circ}$ 3: 1- 566.

Rosen DE. 1964. The relationships and taxonomic position of the halfbeaks, killifishes, silversides and their relatives. Bulletin of the American Museum of Natural History 127: 219-267.

Rosen DE. 1973. Interrelationships of the higher euteleostean Fishes. En: Greenwood PH, RS Miles \& C Patterson (eds). Interrelationships of fishes. Zoological Journal of the Linnean Society 53: 397-513.

Schwindt E. 2003. Arrecifes en la laguna costera Mar Chiquita. Ciencia Hoy 13: 36-41.

Segura V \& JM Díaz de Astarloa. 2004. Análisis osteológico del género Brevoortia (Actinopterygii, Clupeidae) en el Atlántico suroccidental. Revista de Biología Marina y Oceanografía 39: 37-52.

Spivak E, T Luppi \& C Bas. 2001. Cangrejos y camarones: Las relaciones organismo-ambiente en las distintas fases del ciclo de vida. En: Iribarne O. (ed). Reserva de biosfera Mar Chiquita: Características físicas, biológicas y ecológicas, pp. 129-151. Editorial Martín, Mar del Plata.

Vega LE. 2001. Herpetofauna: Diversidad, ecología e historia natural. En: Iribarne O. (ed). Reserva Mar Chiquita: Características físicas, biológicas y ecológicas, pp. 213-226. Editorial Martín, Mar del Plata.

Watt J, GJ Pierce \& PR Boyle. 1997. Guide to the identification of North Sea fish using premaxillae and vertebrae. International Council for the Exploration of the Sea Co-operative Research Report 220: 1-231. 